\title{
PARTIAL GENERALIZED CROSSED PRODUCTS AND A SEVEN-TERM EXACT SEQUENCE
}

\author{
M. DOKUCHAEV, A. PAQUES, H. PINEDO, AND I. ROCHA
}

\begin{abstract}
For a partial Galois extension of commutative rings we give a seven term exact sequence wich generalize the Chase-Harrison-Rosenberg sequence.
\end{abstract}

\section{INTRODUCTION}

This is a continuation of the paper [17], in which a sequence of six homomorphisms was constructed in order to give a partial Galois theoretic analogue of the Chase-HarrisonRosenberg seven term exact sequence [5]. In this article we prove that the sequence from [17] is exact. In some sense this topic is a natural sequel to [13], where a Galois theory for commutative rings was developed in the context of partial actions of groups on rings, including some facts on non-commutative Galois extensions. A systematic algebraic consideration of partial actions was initially influenced by a successful use of partial actions and partial representations in the study of $C^{*}$-algebras generated by partial isometries, and nowadays it is being stimulated by diverse algebraic, topological and $C^{*}$-algebraic advances on the subject (see the book by R. Exel [20] and the surveys [3], [8], [9], [21], $[28])$.

Prior to the sequence, the paper by S.U. Chase, D.K. Harrison, A. Rosenberg [5] contains, in particular, several equivalent definitions of the Auslander-Goldman concept of a Galois extension of commutative rings [1] and a fundamental theorem. This part of [5] was extended in [13] to partial actions, naturally raising the problem of the generalization of the seven term exact sequence as well. Given a (global) Galois extension $R^{G} \subseteq R$ of commutative rings relative to a finite group $G$, the Chase-Harrison-Rosenberg sequence involves Picard groups, Galois cohomology groups and the Auslander-Goldman notion of the Brauer group [1], which in this case is formed by the equivalence classes of Azumaya (i.e. central separable) $R^{G}$-algebras split by $R$. In symbols, the sequence is of

Date: August 19, 2019.

This work was partially supported by FAPESP of Brazil. The first named author was also partially supported by CNPq of Brazil.

Mathematics Subject Classification: Primary 13B05; Secondary 13A50; 16H05; 16K50; 16S35; 16W22; 20M18.

Key words and phrases: Partial action, partial representation, Galois extension, Galois cohomology, Azumaya algebra, Brauer group, Picard group. 
the form

$$
\begin{aligned}
& 0 \rightarrow H^{1}(G, \mathcal{U}(R)) \rightarrow \operatorname{Pic}\left(R^{G}\right) \rightarrow \operatorname{Pic}(R)^{G} \rightarrow H^{2}(G, \mathcal{U}(R)) \rightarrow B\left(R / R^{G}\right) \rightarrow H^{1}(G, \operatorname{Pic}(R)) \rightarrow \\
& H^{3}(G, \mathcal{U}(R)) .
\end{aligned}
$$

Thus for our generalization of the sequence a cohomology theory based on partial group actions is needed. The latter was introduced in [14], inspired by R. Exel's notion of a twisted partial group action on a $C^{*}$-algebra [19], adapted to rings in [11]. The starting point of [14] is the replacement of global $G$-actions on abelian groups ( $G$-modules) by unital partial actions of $G$ on commutative semigroups (partial $G$-modules), in particular, on commutative rings. The partial group cohomology found applications to partial projective group representations [14], [18] and to the study of ideals of (global) reduced $C^{*}$-crossed products [24]. It also motivated the treatment of partial cohomology from the point of view of Hopf algebras [4]. A more general multiplier valued version of partial cohomology was used to classify extensions of semilattices of abelian groups by groups [15], [16].

The action of $G$ on $R$, as above, induces an action of $G$ on the $\operatorname{Picad} \operatorname{group} \operatorname{Pic}(R)$, and the third term of the Chase-Harrison-Rosenberg sequence is the subgroup of the fixed points $\operatorname{Pic}(R)^{G}$. It turns out that a partial action $\alpha$ of $G$ on $R$ gives rise to a partial action $\alpha^{*}$ of $G$ on a more general object, namely the commutative inverse semigroup $\operatorname{PicS}(R)$ of the finitely generated projective $R$-modules of rank $\leq 1$ (see Section 3 ). The semigroup $\operatorname{PicS}(R)$ contains $\operatorname{Pic}(R)$ as a subgroup, and the appropriate replacement of $\mathbf{P i c}(R)^{G}$ is the semigroup of those elements of $\mathbf{P i c}(R)$, which are $\alpha^{*}$-invariant in $\mathbf{P i c S}(R)$. All (global) cohomology groups in the above sequence are substituted now by their partial theoretic analogues, observing that $\mathbf{P i c S}(R)$ appears also in the sixth term as the coefficients of certain 1-cohomologies. Our main result is the following exact sequence:

$$
\begin{aligned}
& 0 \rightarrow H^{1}(G, \alpha, R) \stackrel{\varphi_{1}}{\rightarrow} \mathbf{P i c}\left(R^{\alpha}\right) \stackrel{\varphi_{2}}{\rightarrow} \mathbf{P i c S}(R)^{\alpha^{*}} \cap \mathbf{P i c}(R) \stackrel{\varphi_{3}}{\rightarrow} H^{2}(G, \alpha, R) \stackrel{\varphi_{4}}{\rightarrow} B\left(R / R^{\alpha}\right) \stackrel{\varphi_{5}}{\rightarrow} \\
& H^{1}\left(G, \alpha^{*}, \mathbf{P i c S}(R)\right) \stackrel{\varphi_{6}}{\rightarrow} H^{3}(G, \alpha, R) .
\end{aligned}
$$

The seven term sequence in [5] was derived from the Amitsur cohomology seven term exact sequence, obtained by S.U. Chase and A. Rosenberg in [6] using a spectral sequence of A. Grothendieck. A constructive proof of the Chase-Harrison-Rosenberg sequence was offered by T. Kanzaki in [23], an important ingredient being the notion of a generalized crossed product related to a representation of $G$ into an appropriate Picard group. Our approach is inspired by the treatment of the first six terms of the sequence in the book of F. DeMeyer and E. Ingraham [7] and the Kanzaki's paper [23]. We use an extended version of generalized crossed products, wich in our case are related to a partial representation of $G$ into a certain Picard semigroup (see Section 7).

In this paper by a ring we mean an associative ring with identity element. For any ring $R$, an $R$-module is a left unital $R$-module. If $R$ is commutative, unless otherwise stated, we will consider an $R$-module $M$ as a central $R$-R-bimodule. We say that $M$ is a 
f.g.p. $R$-module if $M$ is a (left) projective and finitely generated $R$-module. A faithfully projective $R$-module is a faithful f.g.p. $R$-module. For a monoid (or a ring) $T$, the group of units of $T$ is denoted by $\mathcal{U}(T)$.

Throughout this work, $R$ will denote a commutative ring and unadorned $\otimes$, unless otherwise established, will mean $\otimes_{R}$.

\section{SOME PRELIMINARIES}

In this section we give some definitions and results which will be used in the paper. We start by recalling three basic facts about projective modules.

Lemma 2.1. The following assertions hold.

- [17, Lemma 2.1] Let $M$ and $N$ be R-modules such that $M$ and $M \otimes N$ are f.g.p. $R$-modules, and $M_{\mathfrak{p}} \neq 0$ for all prime ideals $\mathfrak{p}$ of $R$. Then, $N$ is also a f.g.p. $R$-module.

- [7, Lemma I.3.2 b)] If $M$ is a faithful f.g.p. R-module, then there exists an $R$-R-bimodule isomorphism $M^{*} \otimes_{\operatorname{End}_{R}(M)} M \cong R$. Consequently, if $N$ and $N^{\prime}$ are $R$-modules such that $M \otimes N \cong M \otimes N^{\prime}$ as $R$-modules, then $N \cong N^{\prime}$ as $R$-modules.

- [7, Hom-Tensor Relation I.2.4] Let $A$ and $B$ be R-algebras, $M$ be a f.g.p. Amodule and $N$ be a f.g.p. B-module. Then for any $A$-module $M^{\prime}$ and any $B$ module $N^{\prime}$, the map

$$
\psi: \operatorname{Hom}_{A}\left(M, M^{\prime}\right) \otimes \operatorname{Hom}_{B}\left(N, N^{\prime}\right) \rightarrow \operatorname{Hom}_{(A \otimes B)}\left(M \otimes N, M^{\prime} \otimes N^{\prime}\right),
$$

induced by $(f \otimes g)(m \otimes n)=f(m) \otimes g(n)$, for all $m \in M, n \in N$, is an R-module isomorphism. If $M=M^{\prime}$ and $N=N^{\prime}$, then $\psi$ is an $R$-algebra isomorphism.

We proceed with partial Galois cohomology. Let $G$ be a group. We recall from [11] that a unital twisted partial action of $G$ on $R$ is a triple

$$
\alpha=\left(\left\{D_{g}\right\}_{g \in G},\left\{\alpha_{g}\right\}_{g \in G},\left\{\omega_{g, h}\right\}_{(g, h) \in G \times G}\right),
$$

such that for every $g \in G, D_{g}$ is an ideal of $R$ generated by a non-necessarily non-zero idempotent $1_{g}, \alpha_{g}: D_{g^{-1}} \rightarrow D_{g}$ is an isomorphism of rings, for each pair $(g, h) \in G \times G$, $\omega_{g, h}$ is in $\mathcal{U}\left(D_{g} D_{g h}\right)$, and the following statements are satisfied for all $g, h, l \in G$ :

(i) $D_{1}=R$ and $\alpha_{1}$ is the identity map of $R$,

(ii) $\alpha_{g}\left(D_{g^{-1}} D_{h}\right)=D_{g} D_{g h}$,

(iii) $\alpha_{g} \circ \alpha_{h}(r)=\omega_{g, h} \alpha_{g h}(r) \omega_{g, h}^{-1}$ for any $r \in D_{h^{-1}} D_{(g h)^{-1}}$,

(iv) $\omega_{1, g}=\omega_{g, 1}=1_{g}$ and

(v) $\alpha_{g}\left(1_{g^{-1}} \omega_{h, l}\right) \omega_{g, h l}=\omega_{g, h} \omega_{g h, l}$.

If $R$ is a multiplicative monoid, one obtains from the above definition the concept of a unital twisted partial action of a group on a monoid.

Notice that (v) implies

$$
\alpha_{g}\left(\omega_{g^{-1}, g}\right)=\omega_{g, g^{-1}}, \text { for any } g \in G .
$$


Let $\alpha$ be a unital twisted partial action of $G$ on $R$. The partial crossed product $R \star_{\alpha, \omega} G$ (see [11, Definition 2.2]) is the abelian group $\bigoplus_{g \in G} D_{g} \delta_{g}$, where the $\delta_{g}$ 's are place holder symbols, and the multiplication in $R \star_{\alpha, \omega} G$ is induced by

$$
\left(r_{g} \delta_{g}\right)\left(t_{h} \delta_{h}\right)=r_{g} \alpha_{g}\left(t_{h} 1_{g^{-1}}\right) \omega_{g, h} \delta_{g h}
$$

By [11, Theorem 2.4] $R \star_{\alpha, \omega} G$ is an associative ring with identity $1 \delta_{1}$. Moreover, the map $R \ni r \mapsto r \delta_{1} \in R \star_{\alpha, \omega} G$ is a monomorphism of rings. Thus, we consider $R$ as a subring of $R \star_{\alpha, \omega} G$, and the latter as an $R$ - $R$-bimodule via the actions

$$
r\left(a_{g} \delta_{g}\right)=r a_{g} \delta_{g}, \quad \text { and } \quad\left(a_{g} \delta_{g}\right) r=a_{g} \alpha_{g}\left(r 1_{g^{-1}}\right) \delta_{g}, \quad r \in R, g \in G, a_{g} \in D_{g} .
$$

The family of partial isomorphisms $\left\{\alpha_{g}: D_{g^{-1}} \rightarrow D_{g}\right\}_{g \in G}$ forms a partial action (as defined in [10]), which we also denote by $\alpha$. Then $\omega=\left\{\omega_{g, h}\right\}_{(g, h) \in G \times G}$ is called a twisting of $\alpha$, and the above twisted partial action will be simply denoted by $(\alpha, \omega)$. Moreover, if $\omega(g, h)=1_{g} 1_{g h}$, for all $g, h \in G$, we say that $\omega$ is trivial, and, in this case, the partial crossed product $R \star_{\alpha, \omega} G$ coincides with the partial skew group ring $R \star_{\alpha} G$ as introduced in [10]. The subring of invariants of $R$ under $\alpha$ was defined in [13] as

$$
R^{\alpha}=\left\{r \in R \mid \alpha_{g}\left(r 1_{g^{-1}}\right)=r 1_{g} \forall g \in G\right\} .
$$

When $G$ is finite, the extension $R \supseteq R^{\alpha}$ is a $\alpha$-partial Galois extension (see [13]) if for some $m \in \mathbb{N}$ there exists a subset $\left\{x_{i}, y_{i} \mid 1 \leq i \leq m\right\}$ of $R$, called a partial Galois coordinate system of the extension $R \supseteq R^{\alpha}$, such that $\sum_{i=1}^{m} x_{i} \alpha_{g}\left(y_{i} 1_{g^{-1}}\right)=\delta_{1, g}$, for all $g \in G$.

Remark 2.2. It is shown in [13, Theorem 4.1] that $R \supseteq R^{\alpha}$ is a partial Galois extension if and only if $R$ is a p.f.g $R^{\alpha}$-module and the map

$$
j: R \star_{\alpha} G \rightarrow \operatorname{End}_{R^{\alpha}}(R), \quad j\left(\sum_{g \in G} r_{g} \delta_{g}\right)(r)=\sum_{g \in G} r_{g} \alpha_{g}\left(r 1_{g^{-1}}\right),
$$

for each $r \in R$, is an isomorphism of $R$-modules and $R^{\alpha}$-algebras.

Definition 2.3. [14, Definition 1.4] Let $T$ be a commutative ring or a monoid, $n \in \mathbb{N}$ and $\alpha=\left(T_{g}, \alpha_{g}\right)_{g \in G}$ a unital partial action of $G$ on $T$. An $n$-cochain of $G$ with values in $T$ is a map $f: G^{n} \rightarrow T$, such that $f\left(g_{1}, \ldots, g_{n}\right) \in \mathcal{U}\left(T 1_{g_{1}} 1_{g_{1} g_{2}} \ldots 1_{g_{1} g_{2} \ldots g_{n}}\right)$, for all $n \in \mathbb{N}$. A 0-cochain is an invertible element of $T$.

The set of $n$-cochains $C^{n}(G, \alpha, T)$ is an abelian group under the point-wise multiplication. Its identity is $\left(g_{1}, g_{2} \ldots g_{n}\right) \mapsto 1_{g_{1}} 1_{g_{1} g_{2}} \ldots 1_{g_{1} g_{2} \ldots g_{n}}$ and the inverse of $f \in$ $C^{n}(G, \alpha, T)$ is $f^{-1}\left(g_{1}, \ldots, g_{n}\right)=f\left(g_{1}, \ldots, g_{n}\right)^{-1}$, where $f\left(g_{1}, \ldots, g_{n}\right)^{-1} f\left(g_{1}, \ldots, g_{n}\right)=$ $1_{g_{1}} 1_{g_{1} g_{2}} \ldots 1_{g_{1} g_{2} \ldots g_{n}}$. 
Proposition 2.4. [14, Proposition 1.5] Let $\delta^{n}: C^{n}(G, \alpha, T) \rightarrow C^{n+1}(G, \alpha, T)$ be the map defined by

$$
\begin{aligned}
\left(\delta^{n} f\right)\left(g_{1}, \ldots, g_{n+1}\right)= & \alpha_{g_{1}}\left(f\left(g_{2}, \ldots, g_{n+1}\right) 1_{g_{1}^{-1}}\right) \prod_{i=1}^{n} f\left(g_{1}, \ldots, g_{i} g_{i+1}, \ldots, g_{n+1}\right)^{(-1)^{i}} \\
& f\left(g_{1}, \ldots, g_{n}\right)^{(-1)^{n+1}}
\end{aligned}
$$

for any $f \in C^{n}(G, \alpha, T)$ and $g_{1}, \ldots, g_{n+1} \in G$, where the inverse elements are taken in the corresponding ideals. If $n=0$ and $t \in \mathcal{U}(T)$ we set $\left(\delta^{0} t\right)(g)=\alpha_{g}\left(1_{g^{-1}} t\right) t^{-1}$, for all $g \in G$. Then, $\delta^{n}$ is a homomorphism such that $\left(\delta^{n+1} \delta^{n} f\right)\left(g_{1}, g_{2}, \ldots, g_{n+2}\right)=$ $1_{g_{1}} 1_{g_{1} g_{2}} \ldots 1_{g_{1} g_{2} \ldots g_{n+2}}$, for any $f \in C^{n}(G, \alpha, T)$.

Definition 2.5. The map $\delta^{n}$ is called a coboundary homomorphism. We define the groups $Z^{n}(G, \alpha, T)=\operatorname{ker} \delta^{n}, B^{n}(G, \alpha, T)=\operatorname{im} \delta^{n-1}$ and $H^{n}(G, \alpha, T)=\frac{\operatorname{ker} \delta^{n}}{\operatorname{im} \delta^{n-1}}$ of partial $n$-cocycles, $n$-coboundaries and $n$-cohomologies of $G$ with values in $T, n \geq 1$, respectively. For $n=0$ we set $H^{0}(G, \alpha, T)=Z^{0}(G, \alpha, T)=\operatorname{ker} \delta^{0}$. We say that $f$ and $f^{\prime}$ in $C^{n+1}(G, \alpha, T)$ are cohomologous if $f=\left(\delta^{n} \rho\right) f^{\prime}$, for some $\rho \in C^{n}(G, \alpha, T)$.

From now on $G$ will denote a finite group, $R$ a commutative ring and $R \supseteq R^{\alpha}$ a partial Galois extension.

$$
\begin{gathered}
\text { 3. The EXACT SEQUEnCE } \\
0 \rightarrow H^{1}(G, \alpha, R) \stackrel{\varphi_{1}}{\rightarrow} \mathbf{P i c}\left(R^{\alpha}\right) \stackrel{\varphi_{2}}{\rightarrow} \mathbf{P i c S}(R)^{\alpha^{*}} \cap \mathbf{P i c}(R) \stackrel{\varphi_{3}}{\rightarrow} H^{2}(G, \alpha, R)
\end{gathered}
$$

For any left $R \star_{\alpha} G$-module $M$ we set

$$
M^{G}=\left\{m \in M \mid\left(1_{g} \delta_{g}\right) m=1_{g} m, \text { for all } g \in G\right\} .
$$

Lemma 3.1. For every left $R \star_{\alpha} G$-module $M$ the map $\mu: R \otimes_{R^{\alpha}} M^{G} \rightarrow M$ given by $\mu\left(x \otimes_{R^{\alpha}} m\right)=x m$ is an isomorphism of left $R \star_{\alpha} G$-modules, where $R$ is a left $R \star_{\alpha} G$ module via $\left(r_{g} \delta_{g}\right) \triangleright r=r_{g} \alpha_{g}\left(r 1_{g^{-1}}\right)$.

Proof. By (ii) of [13, Theorem 4.1] the map $\mu$ is an isomorphism of $R$-modules. So, we only must check that $\mu$ is $R \star_{\alpha} G$-linear. For $g \in G, r \in R, r_{g} \in D_{g}$ and $m \in M^{G}$ we have

$$
\begin{aligned}
\mu\left(\left(r_{g} \delta_{g}\right) \triangleright\left(r \otimes_{R^{\alpha}} m\right)\right) & =r_{g} \alpha_{g}\left(r 1_{g^{-1}}\right) m \\
& =\left[r_{g} \alpha_{g}\left(r 1_{g^{-1}}\right)\left(1_{g} \delta_{g}\right)\right] m \\
& =\left[\left(r_{g} \delta_{g}\right)\left(r \delta_{1}\right)\right] m \\
& =\left(r_{g} \delta_{g}\right)\left[\left(r \delta_{1}\right) m\right] \\
& =\left(r_{g} \delta_{g}\right)(r m) \\
& =\left(r_{g} \delta_{g}\right) \mu\left(r \otimes_{R^{\alpha}} m\right),
\end{aligned}
$$

as desired.

We denote by $\mathbf{P i c}\left(R^{\alpha}\right)$ the Picard group of $R^{\alpha}$. For details on the Picard group of a commutative ring the reader may consult [22] or [25]. 
We recall the group homomorphism $\varphi_{1}: H^{1}(G, \alpha, R) \rightarrow \operatorname{Pic}\left(R^{\alpha}\right)$ given in [17]. First of all we have that

$$
\begin{aligned}
& Z^{1}(G, \alpha, R)=\left\{f \in C^{1}(G, \alpha, R) \mid f(g h) 1_{g}=f(g) \alpha_{g}\left(f(h) 1_{g^{-1}}\right), \forall g, h \in G\right\}, \text { and } \\
& B^{1}(G, \alpha, R)=\left\{f \in C^{1}(G, \alpha, R) \mid f(g)=\alpha_{g}\left(a 1_{g^{-1}}\right) a^{-1}, \text { for some } a \in \mathcal{U}(R)\right\} .
\end{aligned}
$$

Let $f \in Z^{1}(G, \alpha, R)$ and let $\theta_{f} \in \operatorname{End}_{R^{\alpha}}\left(R \star_{\alpha} G\right)$ be given by $\theta_{f}\left(r_{g} \delta_{g}\right)=r_{g} f(g) \delta_{g}$ for all $r_{g} \in D_{g}, g \in G$. Then $\theta_{f}$ is an $R^{\alpha}$-algebra homomorphism, and we define a $R \star_{\alpha} G$-module $R_{f}$ by $R_{f}=R$ as sets and with the action of $R \star_{\alpha} G$ induced by

$$
\left(r_{g} \delta_{g}\right) \cdot r=\theta_{f}\left(r_{g} \delta_{g}\right) \triangleright r, \text { for any } r \in R, g \in G .
$$

We know from [17] that $\left[R_{f}^{G}\right] \in \mathbf{P i c}\left(R^{\alpha}\right)$ and the map

$$
\varphi_{1}: H^{1}(G, \alpha, R) \ni \operatorname{cls}(f) \rightarrow\left[R_{f}^{G}\right] \in \mathbf{P i c}\left(R^{\alpha}\right)
$$

is a well defined group homomorphism.

Theorem 3.2. The sequence $0 \rightarrow H^{1}(G, \alpha, R) \stackrel{\varphi_{1}}{\rightarrow} \mathbf{P i c}\left(R^{\alpha}\right)$ is exact.

Proof. If $\varphi_{1}(\operatorname{cls}(f))=\left[R_{f}^{G}\right]=\left[R^{\alpha}\right]$, then by Lemma 3.1 there are $R \star_{\alpha} G$-module isomorphisms $R_{f} \cong R \otimes_{R^{\alpha}} R_{f}^{G} \cong R$. By [17, Remark 2.8] $f$ is normalized, i.e. $f(1)=1$. Let $w \in \operatorname{Hom}_{R \star_{\alpha} G}\left(R_{f}, R\right)$ be an isomorphism. Since $f(1)=1$ we have $w \in \operatorname{Hom}_{R^{\alpha}}(R, R) \cong$ $R \star_{\alpha} G$, by Remark 2.2. Hence, there is an element $W=\sum_{g \in G} r_{g} \delta_{g} \in \mathcal{U}\left(R \star_{\alpha} G\right)$ such that $w(x)=W x$, for all $x \in R$. Recall that $R \star_{\alpha} G$ acts on $R_{f}$ via $V \cdot r=\theta_{f}(V) r, r \in R, V \in$ $R \star_{\alpha} G$. Then,

$$
(V W) x=V(W x)=V(w(x))=w(V \cdot x)=w\left(\theta_{f}(V) x\right)=\left(W \theta_{f}(V)\right) x,
$$

for all $x \in R_{f}=R$, where the third equality above holds because $w \in \operatorname{Hom}_{R \star \alpha} G\left(R_{f}, R\right)$. Thus, $W \theta_{f}(V)=V W$. In particular, if $V=r \delta_{1}=r, \theta_{f}(V)=r$, and this gives $\sum_{g \in G} r_{g} \alpha_{g}\left(r 1_{g^{-1}}\right) \delta_{g}=\sum_{g \in G} r r_{g} \delta_{g}$. Consequently, $j\left(r_{g} \delta_{g}\right)(r)=j\left(r_{g} \delta_{1}\right)(r)$, for all $g \in G$, where $j$ is the isomorphism defined in (2). Therefore, $r_{g}=0$ if $g \neq 1$ and $W=r_{1} \delta_{1} \in$ $\mathcal{U}(R)$. Now, taking $V=1_{h} \delta_{h}$ we get $\theta_{f}(V)=f(h) \delta_{h}$ and

$$
f(h) \delta_{h}=\left(r_{1}^{-1} \delta_{1}\right)\left(1_{h} \delta_{h}\right)\left(r_{1} \delta_{1}\right)=\alpha_{h}\left(r_{1} 1_{h^{-1}}\right) r_{1}^{-1} \delta_{h},
$$

which implies $f \in B^{1}(G, \alpha, R)$.

Let $\kappa$ be a commutative ring and $\Lambda$ be a commutative $\kappa$-algebra with identity. We recall from [17] the following.

Definition 3.3. A non-necessarily central $\Lambda$ - $\Lambda$-bimodule $P$ is called $\kappa$-partially invertible if $P$ is a central $\kappa$-bimodule and as a $\Lambda$ - $\Lambda$-bimodule $P$ satisfies the following two properties.

- $P$ is left and right f.g.p. $\Lambda$-module,

- $\Lambda^{o p} \ni \lambda \mapsto r_{\lambda} \in \operatorname{End}\left({ }_{\Lambda} P\right), r_{\lambda}(p)=p \lambda$ and $\Lambda \ni \lambda \mapsto l_{\lambda} \in \operatorname{End}\left(P_{\Lambda}\right), l_{\lambda}(p)=\lambda p$, are $k$-algebra epimorphisms. 
Let $[P]=\{M \mid M$ is a $\Lambda$ - $\Lambda$-bimodule and $M \cong P$ as $\Lambda$ - $\Lambda$-bimodules $\}$. We denote by $\mathbf{P i c S}_{\kappa}(\Lambda)$ the set of classes $[P]$ of partially invertible $\Lambda$ - $\Lambda$-bimodules with composition $[P][Q]=\left[P \otimes_{\Lambda} Q\right]$. If $\kappa=\Lambda$, we set $\mathbf{P i c S}(\Lambda)=\operatorname{PicS}_{\Lambda}(\Lambda)$.

The following result is [17, Proposition 3.5]. Since the proof presented there has several misprints, we give it below for reader's convenience.

Proposition 3.4. The product $[P][Q]=\left[P \otimes_{\Lambda} Q\right]$ endows $\mathbf{P i c S}_{\kappa}(\Lambda)$ with the structure of a semigroup.

Proof. We shall show that $\left[P \otimes_{\Lambda} Q\right] \in \mathbf{P i c S}_{k}(\Lambda)$, for any $[P],[Q] \in \mathbf{P i c S}_{\kappa}(\Lambda)$. Notice that $P \otimes_{\Lambda} Q$ is a left f.g.p. $\Lambda$-module. Indeed, there are free f.g. left $\Lambda$-modules $F_{1}, F_{2}$ and left $\Lambda$-modules $M_{1}, M_{2}$ such that $P \oplus M_{1}=F_{1}, Q \oplus M_{2}=F_{2}$. Now consider $M_{1}$ and $F_{1}$ as central $\Lambda$ - $\Lambda$-bimodules, then by tensoring the two previous equalities we see that there exists a left $\Lambda$-module $M$ such that $\left(P \otimes_{\Lambda} Q\right) \oplus M \cong F_{1} \otimes_{\Lambda} F_{2}$ as left $\Lambda$-modules, and the assertion follows. In a similar way, one can show that $P \otimes_{\Lambda} Q$ is a right f.g.p. $\Lambda$-module.

By assumption there are $\kappa$-algebra epimorphisms $r^{1}: \Lambda \rightarrow \operatorname{End}\left({ }_{\Lambda} P\right)$ and $r^{2}: \Lambda \rightarrow$ $\operatorname{End}\left({ }_{\Lambda} Q\right)$, given by right multiplications. It follows, using the third item of Lemma 2.1, that

$$
r^{1} \otimes r^{2}: \Lambda \otimes_{\Lambda} \Lambda \rightarrow \operatorname{End}\left({ }_{\Lambda} P\right) \otimes_{\Lambda} \operatorname{End}\left({ }_{\Lambda} Q\right) \cong \operatorname{End}_{\Lambda}\left(P \otimes_{\Lambda} Q\right)
$$

is a $\kappa$-algebra epimorphism. Since $\Lambda \ni \lambda \mapsto 1_{\Lambda} \otimes_{\Lambda} \lambda \in \Lambda \otimes_{\Lambda} \Lambda$ is a $\kappa$-algebra isomorphism, we conclude that $\Lambda \ni \lambda \mapsto r_{1_{\Lambda}}^{1} \otimes r_{\lambda}^{2} \in \operatorname{End}_{\Lambda}\left(P \otimes_{\Lambda} Q\right)$ is a $\kappa$-algebra epimorphism. Now for any $p \otimes_{\Lambda} q \in P \otimes_{\Lambda} Q$, we have that $r_{1_{\Lambda}}^{1} \otimes r_{\lambda}^{2}\left(p \otimes_{\Lambda} q\right)=p \otimes_{\Lambda} q \lambda$, and the assertion follows. In an analogous way we obtain that the left multiplication is a $\kappa$-algebra epimorphism.

Definition 3.5. [17, Definition 3.1] We say that a f.g.p. central R-R-bimodule $P$ has rank less than or equal to one, if for any $\mathfrak{p} \in \operatorname{Spec}(R)$ one has $P_{\mathfrak{p}}=0$ or $P_{\mathfrak{p}} \cong R_{\mathfrak{p}}$ as $R_{\mathfrak{p}}$-modules. In this case we write $\operatorname{rk}_{R}(P) \leq 1$.

We remind a characterization of $\mathbf{P i c S}(R)$.

Proposition 3.6. For any commutative ring $R$ we have

- $[17$, Definition 3.1, Remark 3.4]

$$
\operatorname{PicS}(R)=\{[E] \mid E \text { is a f.g.p. R-module and } \operatorname{rk}(E) \leq 1\} \text {. }
$$

- $[17$, Proposition 3.8, arXiv version]

The set $\mathbf{P i c S}(R)$, with binary operation induced by the tensor product, is a commutative inverse monoid with 0. Moreover, the inverse $[E]^{*}$ of $[E]$ is $\left[E^{*}\right]$, where $E^{*}=\operatorname{hom}_{R}(E, R)$ is the dual of $E$.

- $[17$, Formula (3.1)]

$$
\operatorname{PicS}(R) \cong \bigcup_{e \in \mathbf{I}_{\mathbf{p}}(R)} \operatorname{Pic}(R e),
$$

where $\mathbf{I}_{\mathbf{p}}(R)$ denotes the semilattice of the idempotents of $R$ with respect to the product. 
We recall also the next.

Lemma 3.7. [17, Lemma 3.12, arXiv version] Let $g \in G$ and $[M] \in \mathbf{P i c S}(R)$. If $1_{g} m=$ $m$, for all $m \in M$ and $M_{\mathfrak{p}} \cong\left(D_{g}\right)_{\mathfrak{p}}$ as $R_{\mathfrak{p}}$-modules, for all $\mathfrak{p} \in \operatorname{Spec}(R)$, then $[M] \in$ $\operatorname{Pic}\left(D_{g}\right)$.

For the sequel, we proceed by recalling a partial action of $G$ on $\operatorname{PicS}(R)$ given in [17]. Let $\alpha=\left(D_{g}, \alpha_{g}\right)_{g \in G}$ be a partial action of $G$ on $R$. Then, for any $y \in R$ we have

$$
\alpha_{g}\left(\alpha_{h}\left(y 1_{h^{-1}}\right) 1_{g^{-1}}\right)=\alpha_{g h}\left(y 1_{(g h)^{-1}}\right) 1_{g}, \quad \text { for all } g, h \in G .
$$

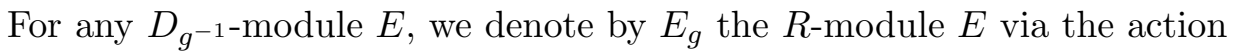

$$
r \bullet x=\alpha_{g^{-1}}\left(r 1_{g}\right) x,
$$

for any $r \in R, x \in E$.

Lemma 3.8. We have the following.

- $[17$, Lemma 3.6, vi $)]$ For any $[M] \in \mathbf{P i c}\left(D_{g^{-1}}\right),\left[M_{g}\right] \in \mathbf{P i c}\left(D_{g}\right)$.

- $[17$, Lemma 3.7 $] X_{g}=\left[D_{g}\right] \mathbf{P i c S}(R)=\left\{[E] \in \mathbf{P i c S}(R) \mid E=1_{g} E\right\}$.

- [17, Theorem 3.8] The family $\alpha^{*}=\left(X_{g}, \alpha_{g}^{*}\right)_{g \in G}$, where

$$
\alpha_{g}^{*}: X_{g^{-1}} \ni[E] \mapsto\left[E_{g}\right] \in X_{g},
$$

gives a partial action of $G$ on the inverse semigroup $\mathbf{P i c S}(R)$. Moreover, $\mathcal{U}\left(X_{g}\right)=$ $\operatorname{Pic}\left(D_{g}\right)$.

- $[17$, Proposition 4.5, arXiv version]

$$
\operatorname{PicS}(R)^{\alpha^{*}}=\left\{[E] \in \mathbf{P i c S}(R) \mid\left(E \otimes D_{g^{-1}}\right)_{g} \cong E \otimes D_{g}, \text { for allg } \in G\right\}
$$

has a 0 and is a commutative inverse submonoid of $\mathbf{P i c S}(R)$. Moreover,

$$
\left[\left(E^{*}\right)_{g}\right]=\left[E_{g}\right]^{*}=\left[\left(E_{g}\right)^{*}\right],
$$

for any $g \in G$.

Proposition 3.9. [17, Proposition 4.2] The map

$$
\varphi_{2}: \operatorname{Pic}\left(R^{\alpha}\right) \ni[E] \mapsto\left[R \otimes_{R^{\alpha}} E\right] \in \mathbf{P i c S}(R)^{\alpha^{*}} \cap \operatorname{Pic}(R),
$$

is a group homomorphism.

Theorem 3.10. The sequence $H^{1}(G, \alpha, R) \stackrel{\varphi_{1}}{\rightarrow} \mathbf{P i c}\left(R^{\alpha}\right) \stackrel{\varphi_{2}}{\rightarrow} \mathbf{P i c S}(R)^{\alpha^{*}} \cap \mathbf{P i c}(R)$ is exact.

Proof. Let $f \in Z^{1}(G, \alpha, R)$. Then $\varphi_{2} \varphi_{1}(\operatorname{cls}(f))=\left[R \otimes_{R^{\alpha}} R_{f}^{G}\right]$ and $R \otimes_{R^{\alpha}} R_{f}^{G} \cong$ $R_{f}$, the latter being the isomorphism of $R \star_{\alpha} G$-modules given by Lemma 3.1. Thus, $R \otimes_{R^{\alpha}} R_{f}^{G} \cong R$ as $R$-modules, and $\operatorname{im} \varphi_{1} \subseteq \operatorname{ker} \varphi_{2}$.

Let $[M] \in \operatorname{Pic}\left(R^{\alpha}\right)$ and assume $[M] \in \operatorname{ker} \varphi_{2}$. Then, there is an $R$-module isomorphism $\xi: R \otimes_{R^{\alpha}} M \rightarrow R$. Observe that $\xi\left(D_{g} \otimes_{R^{\alpha}} M\right)=1_{g} \xi\left(R \otimes_{R^{\alpha}} M\right)=1_{g} R=D_{g}$. Since $R$ is an $R \star_{\alpha} G$-module, with action $\triangleright$ defined in Lemma $3.1, R \otimes_{R^{\alpha}} M$ is also an $R \star_{\alpha} G$-module 
by letting $R \star_{\alpha} G$ act on $R$. We identify $R \star_{\alpha} G$ with $\operatorname{End}_{R^{\alpha}}(R)$ via $(2)$ and define a map $\theta \in \operatorname{End}_{R^{\alpha}}\left(R \star_{\alpha} G\right)$ by

$$
\theta(W)(x)=\xi\left(W \triangleright \xi^{-1}(x)\right), \text { for all } W \in R \star_{\alpha} G \text { and } x \in R .
$$

Notice that $\theta$ is an $R^{\alpha}$-algebra homomorphism and $\theta(r)(x)=r x$ for all $r, x \in R$.

Let $f: G \ni g \mapsto \theta\left(1_{g} \delta_{g}\right)(1) \in R$, where $\theta\left(1_{g} \delta_{g}\right)$ is viewed as an element of $\operatorname{End}_{R^{\alpha}}(R)$. We shall prove that $f \in Z^{1}(G, \alpha, R)$.

First of all, since $1_{g} \delta_{g} \triangleright \xi^{-1}(1) \in D_{g} \otimes_{R^{\alpha}} M$ we see that $f(g) \in D_{g}$, for all $g \in G$. On the other hand,

$$
\theta\left(1_{g} \delta_{g}\right)(r)=\xi\left(1_{g} \delta_{g} \triangleright \xi^{-1}(r)\right)=\xi\left(\alpha_{g}\left(r 1_{g^{-1}}\right) \delta_{g} \triangleright \xi^{-1}(1)\right)=\alpha_{g}\left(r 1_{g^{-1}}\right) \xi\left(1_{g} \delta_{g} \triangleright \xi^{-1}(1)\right),
$$

and so

$$
\theta\left(1_{g} \delta_{g}\right)(r)=\alpha_{g}\left(r 1_{g^{-1}}\right) f(g), \quad \text { for each } g \in G \text { and } r \in R
$$

In particular, for $r_{g}=\theta\left(1_{g^{-1}} \delta_{g^{-1}}\right)\left(1_{g}\right)$, we have

$$
\begin{aligned}
\alpha_{g}\left(r_{g} 1_{g^{-1}}\right) f(g) & =\theta\left(1_{g} \delta_{g}\right)\left(\theta\left(1_{g^{-1}} \delta_{g^{-1}}\right)\left(1_{g}\right)\right) \\
& =\left[\theta\left(1_{g} \delta_{g}\right) \theta\left(1_{g^{-1}} \delta_{g^{-1}}\right)\right]\left(1_{g}\right) \\
& =\theta\left(1_{g} \delta_{1}\right)\left(1_{g}\right) \\
& =1_{g},
\end{aligned}
$$

ensuring that $f(g) \in \mathcal{U}\left(D_{g}\right)$, for all $g \in G$. Thus, $f \in C^{1}(G, \alpha, R)$.

Now we show that $f$ satisfies the $(1, \alpha)$-cocycle identity. By $(8)$ we have $\theta\left(1_{g} \delta_{g}\right)(r)=$ $\left(f(g) \delta_{g}\right)(r)$, for any $r \in R$. Then, $\theta\left(1_{g} \delta_{g}\right)=f(g) \delta_{g}$ as maps defined on $R$, and $f(g) \alpha_{g}\left(f(h) 1_{g^{-1}}\right) \delta_{g h}=\left(f(g) \delta_{g}\right)\left(f(h) \delta_{h}\right)=\theta\left(1_{g} \delta_{g}\right) \theta\left(1_{h} \delta_{h}\right)=1_{g} \theta\left(1_{g h} \delta_{g h}\right)=1_{g} f(g h) \delta_{g h}$, showing that $f \in Z^{1}(G, \alpha, R)$. To end the proof we must show that there is an $R^{\alpha}$ module isomorphism from $M$ to $R_{f}^{G}$. Consider the map $\xi^{-1}: R_{f} \rightarrow R \otimes_{R^{\alpha}} M$, then for each $z \in R_{f}$ we have

$$
\xi^{-1}\left(\left(r_{g} \delta_{g}\right) \cdot z\right) \stackrel{(3)}{=} \xi^{-1}\left(r_{g} \alpha_{g}\left(z 1_{g^{-1}}\right) f(g)\right) \stackrel{(8)}{=} \xi^{-1}\left(\theta\left(r_{g} \delta_{g}\right)(z)\right) \stackrel{(7)}{=}\left(r_{g} \delta_{g}\right) \triangleright \xi^{-1}(z) .
$$

Hence, there are isomorphisms of $R \star_{\alpha} G$-modules $R \otimes_{R^{\alpha}} R_{f}^{G} \cong R_{f} \cong R \otimes_{R^{\alpha}} M$, which leads to $R_{f}^{G} \cong M$ as $R^{\alpha}$-modules, thanks to the second item of Lemma 2.1. Therefore, $[M]=\varphi_{1}(\operatorname{cls}(f))$.

We recall from [17] the homomorphism $\varphi_{3}: \operatorname{PicS}(R)^{\alpha^{*}} \cap \operatorname{Pic}(R) \rightarrow H^{2}(G, \alpha, R)$. First of all we have that $B^{2}(G, \alpha, R)$ is the group

$$
\left\{w \in C^{2}(G, \alpha, R) \mid \exists f \in C^{1}(G, \alpha, R) \text {, with } w(g, h)=\alpha_{g}\left(f(h) 1_{g^{-1}}\right) f(g) f(g h)^{-1}\right\} .
$$

and $Z^{2}(G, \alpha, R)$ is

$$
\left\{w \in C^{2}(G, \alpha, R) \mid \alpha_{g}\left(w(h, l) 1_{g^{-1}}\right) w(g, h l)=w(g h, l) w(g, h), \forall g, h, l \in G\right\} .
$$

Now, let $[E]$ be an element of $\mathbf{P i c S}(R)^{\alpha^{*}} \cap \operatorname{Pic}(R)$. Then, there is a family of $R$-module isomorphisms

$$
\left\{\psi_{g}: E D_{g} \rightarrow\left(E D_{g^{-1}}\right)_{g}\right\}_{g \in G}, \text { with } \psi_{g}(r x)=\alpha_{g^{-1}}\left(r 1_{g}\right) \psi_{g}(x),
$$


for all $r \in R, x \in E D_{g}, g \in G,{ }^{1}$ and the maps, $\psi_{g}^{-1}:\left(E D_{g^{-1}}\right)_{g} \rightarrow E D_{g}, g \in G$, satisfy

$$
\psi_{g}^{-1}(r x)=\alpha_{g}\left(r 1_{g^{-1}}\right) \psi_{g}^{-1}(x), \text { for all } g \in G, r \in R, x=x_{g^{-1}} \in E D_{g^{-1}} .
$$

Hence, $\psi_{(g h)^{-1}} \psi_{h^{-1}}^{-1} \psi_{g^{-1}}^{-1}: E D_{g} D_{g h} \rightarrow E D_{g} D_{g h}$ belongs to $\mathcal{U}\left(\operatorname{End}_{D_{g} D_{g h}}\left(E D_{g} D_{g h}\right)\right)$. Since $[E] \in \mathbf{P i c}(R)$, it follows that $\left[E D_{g} D_{g h}\right] \in \mathbf{P i c}\left(D_{g} D_{g h}\right)$, and consequently we have $\operatorname{End}_{D_{g} D_{g h}}\left(E D_{g} D_{g h}\right) \cong D_{g} D_{g h}$. Therefore, there exists $\omega_{g, h} \in \mathcal{U}\left(D_{g} D_{g h}\right)$ such that

$$
\psi_{(g h)^{-1}} \psi_{h^{-1}}^{-1} \psi_{g^{-1}}^{-1}(x)=\omega_{g, h} x, \text { for all } x \in E D_{g} D_{g h} .
$$

Summarizing, for an element $[E] \in \mathbf{P i c S}(R)^{\alpha^{*}} \cap \mathbf{P i c}(R)$ we have found a map $\omega_{[E]}=$ $\omega: G \times G \ni(g, h) \mapsto \omega_{g, h} \in \mathcal{U}\left(D_{g} D_{g h}\right) \subseteq R$. Moreover, $\omega \in Z^{2}(G, \alpha, R)$ and by $[17$, Claim 4.3] $\operatorname{cls}(\omega)$ does not depend on the choice of the family of isomorphisms given by (9).

Proposition 3.11. $\left[17\right.$, Claim 4.4] $\varphi_{3}: \operatorname{PicS}(R)^{\alpha^{*}} \cap \operatorname{Pic}(R) \ni[E] \mapsto \operatorname{cls}(\omega) \in H^{2}(G, \alpha, R)$ is a group homomorphism.

We need the following.

Lemma 3.12. Let $[E] \in \operatorname{PicS}(R)^{\alpha^{*}}$ and $\left\{\psi_{g}: E D_{g} \rightarrow\left(E D_{g^{-1}}\right)_{g}\right\}_{g \in G}$ be a family of $R$-module isomorphisms with $\psi_{1}=\mathrm{id}_{E}$. Suppose that $c_{g}$ are fixed elements in $D_{g}, g \in G$, with $c_{1}=1$. Then the action

$$
\left(\sum_{g \in G} a_{g} \delta_{g}\right) x=\sum_{g \in G} a_{g} c_{g} \psi_{g^{-1}}\left(x 1_{g^{-1}}\right)
$$

endows the $R$-module $E$ with an $R \star_{\alpha, \omega} G$-module structure, provided that

$$
\omega_{g, h} c_{g h} \psi_{(g h)^{-1}}(x)=c_{g} \alpha_{g}\left(c_{h} 1_{g^{-1}}\right) \psi_{g^{-1}} \psi_{h^{-1}}(x)
$$

holds for all $g, h \in G$ and $x \in E D_{h^{-1}} D_{(g h)^{-1}}$.

Proof. Since $\psi_{1}=\operatorname{id}_{E}$ and the action is linear we only need to check the equality $\left[\left(a_{g} \delta_{g}\right)\left(a_{h} \delta_{h}\right)\right] x=\left(a_{g} \delta_{g}\right)\left[\left(a_{h} \delta_{h}\right) x\right]$ for all $g, h \in G$ and $x \in E$. From (10) we obtain that

$$
\psi_{g^{-1}}\left(\psi_{h^{-1}}\left(x 1_{h^{-1}}\right) 1_{g^{-1}}\right)=\psi_{g^{-1}}\left(\psi_{h^{-1}}\left(x 1_{(g h)^{-1}} 1_{h^{-1}}\right)\right) \text { for all } g, h \in G, x \in E .
$$

Then

$$
\begin{aligned}
{\left[\left(a_{g} \delta_{g}\right)\left(a_{h} \delta_{h}\right)\right] x } & =a_{g} \alpha_{g}\left(a_{h} 1_{g^{-1}}\right) \omega_{g, h} c_{g h} \psi_{(g h)^{-1}}\left(x 1_{(g h)^{-1}}\right) \\
& =a_{g} \alpha_{g}\left(a_{h} 1_{g^{-1}}\right) \omega_{g, h} 1_{g} 1_{g h} c_{g h} \psi_{(g h)^{-1}}\left(x 1_{(g h)^{-1}}\right) \\
& =a_{g} \alpha_{g}\left(a_{h} 1_{g^{-1}}\right) \omega_{g, h} c_{g h} \psi_{(g h)^{-1}}\left(x 1_{(g h)^{-1}}\right) \alpha_{g h}\left(1_{h^{-1}} 1_{(g h)^{-1}}\right) \\
& =a_{g} \alpha_{g}\left(a_{h} 1_{g^{-1}}\right) \omega_{g, h} c_{g h} \psi_{(g h)^{-1}}\left(x 1_{(g h)^{-1}} 1_{h^{-1}}\right)
\end{aligned}
$$

\footnotetext{
${ }^{1}$ Since $E \otimes D_{g} \ni x \otimes d \mapsto x d \in E D_{g}$ is a $R$-module isomorphism, we identify $E \otimes D_{g}$ with $E D_{g}$, for any $g \in G$.
} 
On the other hand

$$
\begin{aligned}
\left(a_{g} \delta_{g}\right)\left[\left(a_{h} \delta_{h}\right) x\right] & =\left(a_{g} \delta_{g}\right)\left(a_{h} c_{h} \psi_{h^{-1}}\left(x 1_{h^{-1}}\right)\right) \\
& =a_{g} c_{g} \psi_{g^{-1}}\left(a_{h} c_{h} \psi_{h^{-1}}\left(x 1_{h^{-1}}\right) 1_{g^{-1}}\right) \\
& =a_{g} c_{g} \alpha_{g}\left(a_{h} c_{h} 1_{g^{-1}}\right) \psi_{g^{-1}}\left(\psi_{h^{-1}}\left(x 1_{h^{-1}}\right) 1_{g^{-1}}\right) \\
& \stackrel{(12)}{=} a_{g} \alpha_{g}\left(a_{h} 1_{g^{-1}}\right) c_{g} \alpha_{g}\left(c_{h} 1_{g^{-1}}\right) \psi_{g^{-1}} \psi_{h^{-1}}\left(x 1_{(g h)^{-1}} 1_{h^{-1}}\right) .
\end{aligned}
$$

Using (11) the Lemma follows.

Theorem 3.13. The sequence $\mathbf{P i c}\left(R^{\alpha}\right) \stackrel{\varphi_{2}}{\rightarrow} \operatorname{PicS}(R)^{\alpha^{*}} \cap \operatorname{Pic}(R) \stackrel{\varphi_{3}}{\rightarrow} H^{2}(G, \alpha, R)$ is exact.

Proof. If $[E] \in \mathbf{P i c}\left(R^{\alpha}\right)$, then $\varphi_{2}([E])=\left[R \otimes_{R^{\alpha}} E\right]$ by the definition of $\varphi_{2}$. There are isomorphisms $\left(R \otimes_{R^{\alpha}} E\right) \otimes D_{g} \cong D_{g} \otimes_{R^{\alpha}} E$ and $\left(\left(R \otimes_{R^{\alpha}} E\right) \otimes D_{g^{-1}}\right)_{g} \cong\left(D_{g^{-1}} \otimes_{R^{\alpha}} E\right)_{g}$, of $R$-modules. Notice that $D_{g} \otimes_{R^{\alpha}} E \cong\left(D_{g^{-1}} \otimes_{R^{\alpha}} E\right)_{g}$ as $R$-modules via the map $d \otimes_{R^{\alpha}} x \stackrel{\psi_{g}}{\mapsto}$ $\alpha_{g^{-1}}(d) \otimes_{R^{\alpha}}$, where $x, d \in D_{g}, x \in E, g \in G$. Then $\psi_{(g h)^{-1}} \psi_{h^{-1}}^{-1} \psi_{g^{-1}}^{-1}(d x)=1_{g} 1_{g h}(d x)$, for all $d \in D_{g} D_{g h}$ and any $x \in E, g, h \in G$. Therefore $\operatorname{im} \varphi_{2} \subseteq \operatorname{ker} \varphi_{3}$.

Let $[V] \in \operatorname{ker} \varphi_{3}$. Then $\varphi_{3}([V])=\operatorname{cls}(\omega)$, with $\omega \in B^{2}(G, \alpha, R)$. There are $R$-module isomorphisms $\psi_{g}: V D_{g} \rightarrow\left(V D_{g^{-1}}\right)_{g}$ and a map $u: G \ni g \rightarrow u_{g} \in \mathcal{U}\left(D_{g}\right) \subseteq R$ such that

$$
\psi_{(g h)^{-1}} \psi_{h^{-1}}^{-1} \psi_{g^{-1}}^{-1}(x)=\omega_{g, h} x=u_{g} \alpha_{g}\left(u_{h} 1_{g^{-1}}\right) u_{g h}^{-1} x, \quad x \in V D_{g} D_{g h}, \quad g, h \in G .
$$

Since $\operatorname{cls}(\omega)$ does not depend on the choice of the isomorphisms, we can choose $\psi_{1}=\mathrm{id}_{V}$. Lemma 3.12 and (9) imply that we can view $V$ as an $R \star_{\alpha} G$-module via the action

$$
\left(a_{g} \delta_{g}\right) x=a_{g} u_{g} \psi_{g^{-1}}\left(x 1_{g^{-1}}\right),
$$

for all $a_{g} \in D_{g}, x \in V$ and $g \in G$. By Lemma 3.1 there is an $R \star_{\alpha} G$-module isomorphism $V \cong R \otimes_{R^{\alpha}} V^{G}$. Notice that $V$ is a f.g.p. $R^{\alpha}$-module, therefore by the first item of Lemma 2.1, $V^{G}$ is a f.g.p. $R^{\alpha}$-module. Hence, it only remains to prove that $\left[V^{G}\right] \in \operatorname{Pic}\left(R^{\alpha}\right)$, in order to show that $V \in \operatorname{im} \varphi_{2}$. Let $\mathfrak{p} \in \operatorname{Spec}\left(R^{\alpha}\right)$. The fact that $R$ is a f.g.p. $R^{\alpha}-$ module and [26, Proposition 20.6] imply that $R_{\mathfrak{p}}$ is a semi-local ring, and, consequently $\operatorname{Pic}\left(R_{\mathfrak{p}}\right)=0$ (see [25, Example $\left.2.22(\mathrm{D})\right]$ ) which leads to $V_{\mathfrak{p}} \cong R_{\mathfrak{p}}$ as $R_{\mathfrak{p}}$-modules. Thus, $R_{\mathfrak{p}} \otimes_{\left(R^{\alpha}\right)_{\mathfrak{p}}}\left(R^{\alpha}\right)_{\mathfrak{p}} \cong R_{\mathfrak{p}} \cong V_{\mathfrak{p}} \cong R_{\mathfrak{p}} \otimes_{\left(R^{\alpha}\right)_{\mathfrak{p}}}\left(V^{G}\right)_{\mathfrak{p}}$ as $\left(R^{\alpha}\right)_{\mathfrak{p}}$-modules. The required follows now from the the second item of Lemma 2.1.

\section{The homomorphism $\varphi_{4}$ And EXactness at $H^{2}(G, \alpha, R)$}

Proposition 4.1. $\left[17\right.$, Theorem 5.7] The map $\varphi_{4}: H^{2}(G, \alpha, R) \ni \operatorname{cls}(\omega) \mapsto\left[R \star_{\alpha, \omega} G\right] \in$ $B\left(R / R^{\alpha}\right)$ is a group homomorphism.

Theorem 4.2. The sequence $\operatorname{PicS}(R)^{\alpha^{*}} \cap \operatorname{Pic}(R) \stackrel{\varphi_{3}}{\rightarrow} H^{2}(G, \alpha, R) \stackrel{\varphi_{4}}{\rightarrow} B\left(R / R^{\alpha}\right)$ is exact.

Proof. Let $[E]$ be an element of $\operatorname{PicS}(R)^{\alpha^{*}} \cap \operatorname{Pic}(R)$ and write $\varphi_{3}([E])=\operatorname{cls}(\omega)$. Let $\left\{\psi_{g}: E D_{g} \rightarrow\left(E D_{g^{-1}}\right)_{g} \mid g \in G\right\}$, (where $\psi_{1}=\operatorname{id}_{E}$ ) be a family of $R$-module isomorphisms determining $\omega$. That is, $\omega_{g, h} x=\psi_{(g h)^{-1}} \psi_{h^{-1}}^{-1} \psi_{g^{-1}}^{-1} x$, for all $g, h \in G$ and all $x \in E D_{g} D_{g h}$. Then, it is immediate to see that (11), with $c_{g}=1_{g}$ and $\omega$ replaced 
by $\omega^{-1}$, is satisfied, and by Lemma 3.12 we may consider $E$ as an $R \star_{\alpha, \omega^{-1}} G$-module, where the action of $R \star_{\alpha, \omega^{-1}} G$ on $E$ is induced by

$$
\left(a_{g} \delta_{g}\right) x=a_{g} \psi_{g^{-1}}\left(x 1_{g^{-1}}\right), x \in E, g \in G, a_{g} \in D_{g}
$$

(Notice that with this new action the structure of $E$ as an $R$-module does not change.) Hence, there is an $R^{\alpha}$-algebra homomorphism $\zeta: R \star_{\alpha, \omega^{-1}} G \rightarrow \operatorname{End}_{R^{\alpha}}(E), \zeta(W)(x)=$ $W x$, for all $W \in R \star_{\alpha, \omega^{-1}} G$ and $x \in E$. We shall prove that $\zeta$ is an isomorphism. Set $C=C_{\operatorname{End}_{R^{\alpha}}(E)}\left(R \star_{\alpha, \omega^{-1}} G\right)$. By [7, Theorem 2.4.3] there is an $R^{\alpha}$-algebra isomorphism

$$
R \star_{\alpha, \omega^{-1}} G \otimes_{R^{\alpha}} C \cong \operatorname{End}_{R^{\alpha}}(E)
$$

given by multiplication. Since $R \star_{\alpha, \omega^{-1}} G$ and $\operatorname{End}_{R^{\alpha}}(E)$ are f.g.p. $R^{\alpha}$-modules and $\left(R \star_{\alpha, \omega^{-1}} G\right)_{\mathfrak{p}} \neq 0$ for all $\mathfrak{p} \in \operatorname{Spec}\left(R^{\alpha}\right)$, the first item of Lemma 2.1 and (13) imply that $C$ is a f.g.p. $R^{\alpha}$-module. Now, for any $\mathfrak{p} \in \operatorname{Spec}\left(R^{\alpha}\right)$, the ring $R_{\mathfrak{p}}$ is semilocal and $E_{\mathfrak{p}} \cong R_{\mathfrak{p}}$ as $R_{\mathfrak{p}}$-modules, because $[E] \in \mathbf{P i c}(R)$. Thus, as $R_{\mathfrak{p}}^{\alpha}$-modules $\operatorname{End}_{R^{\alpha}}(E)_{\mathfrak{p}} \cong$ $\operatorname{End}_{R^{\alpha}}(R)_{\mathfrak{p}} \cong\left(R \star_{\alpha, \omega^{-1}} G\right)_{\mathfrak{p}}$, where the latter isomorphism is $j_{\mathfrak{p}}$ induced by (2). Then, there exists $n_{\mathfrak{p}} \in \mathbb{N}$ such that $\operatorname{End}_{R^{\alpha}}(E)_{\mathfrak{p}} \cong\left(R \star_{\alpha, \omega^{-1}} G\right)_{\mathfrak{p}} \cong\left(R^{\alpha}\right)_{\mathfrak{p}}^{n_{\mathfrak{p}}}$, and we conclude from (13) that $\operatorname{rk}_{R^{\alpha}}(C)=1$. Then there exists $u_{\mathfrak{p}} \in C_{\mathfrak{p}}$ such that $C_{\mathfrak{p}}=\left(R^{\alpha}\right)_{\mathfrak{p}} u_{\mathfrak{p}}$ and localizing (13) we have

$$
\operatorname{End}_{R^{\alpha}}(E)_{\mathfrak{p}}=\left(R \star_{\alpha, \omega^{-1}} G\right)_{\mathfrak{p}} C_{\mathfrak{p}}=\left(R \star_{\alpha, \omega^{-1}} G\right)_{\mathfrak{p}}\left(R^{\alpha}\right)_{\mathfrak{p}} u_{\mathfrak{p}}=\left(R \star_{\alpha, \omega^{-1}} G\right)_{\mathfrak{p}} u_{\mathfrak{p}},
$$

from which we see that $u_{\mathfrak{p}}$ is a unit in $\operatorname{End}_{R^{\alpha}}(E)_{\mathfrak{p}}$ and $\operatorname{End}_{R^{\alpha}}(E)_{\mathfrak{p}}=\left(R \star_{\alpha, \omega^{-1}} G\right)_{\mathfrak{p}}$. Since $\mathfrak{p}$ is arbitrary and $\operatorname{End}_{R^{\alpha}}(E) \supseteq R \star_{\alpha, \omega^{-1}} G$, then $\operatorname{End}_{R^{\alpha}}(E)=R \star_{\alpha, \omega^{-1}} G$. Furthermore, $E$ is a faithfully projective $R^{\alpha}$-module and we get $\left[R \star_{\alpha, \omega^{-1}} G\right]=\left[\operatorname{End}_{R^{\alpha}}(E)\right]=\left[R^{\alpha}\right]$. Then $\operatorname{cls}\left(\omega^{-1}\right) \in \operatorname{ker} \varphi_{4}$, and, consequently $\operatorname{cls}(\omega) \in \operatorname{ker} \varphi_{4}$. Hence $\operatorname{im} \varphi_{3} \subseteq \operatorname{ker} \varphi_{4}$.

To prove the converse inclusion take $\operatorname{cls}(\omega) \in \operatorname{ker} \varphi_{4}$. Then $\operatorname{cls}\left(\omega^{-1}\right) \in \operatorname{ker} \varphi_{4}$ and thus $\left[R \star_{\alpha, \omega^{-1}} G\right]=\left[R^{\alpha}\right]$. By [1, Prop. 5.3] there exists a faithfully projective $R^{\alpha}$-module $V$ and a $R^{\alpha}$-algebra isomorphism $\Upsilon: R \star_{\alpha, \omega^{-1}} G \rightarrow \operatorname{End}_{R^{\alpha}}(V)$, and we may view $V$ as a (faithful) $R \star_{\alpha, \omega^{-1}} G$-module (and, consequently, as a faithful and central $R$-bimodule) via $W \diamond v=\Upsilon(W)(v)$.

Recall that $R$ is a maximal commutative $R^{\alpha}$-subalgebra in $R \star_{\alpha, \omega^{-1}} G$ (see [27, Lemma $2.1 \mathrm{vi}$ ), Prop. 3.2]). We may consider a copy of $R$ in $\operatorname{End}_{R^{\alpha}}(V)$ via the identification $R=\Upsilon\left(R \delta_{1}\right)$. Then $\operatorname{End}_{R^{\alpha}}(V)$ is an Azumaya $R^{\alpha}$-algebra and $R=\Upsilon\left(R \delta_{1}\right)$ is a maximal commutative $R^{\alpha}$-subalgebra of $\operatorname{End}_{R^{\alpha}}(V)$. Since $R \supseteq R^{\alpha}$ is separable, [1, Theorem 5.6] implies that $\operatorname{End}_{R^{\alpha}}(V)$ is f.g.p. $R$-module. Moreover, by [2, Theorem 2] $V$ is a right f.g.p. $\operatorname{End}_{R^{\alpha}}(V)$-module, and we see that $V$ is a f.g.p. $R$-module. Furthermore, since $\operatorname{End}_{R}(V)=C_{\operatorname{End}_{R^{\alpha}}(V)}(R)=C_{R \star_{\alpha, \omega}-1} G(R)=R$, we conclude that $[V] \in \mathbf{P i c}(R)$.

We show that $[V]$ is an element of $\operatorname{PicS}(R)^{\alpha^{*}}$. First notice that the map

$$
V \times D_{g} \ni(v, d) \mapsto\left(1_{g^{-1}} \delta_{g^{-1}} \diamond v\right) \otimes \alpha_{g^{-1}}(d) \in\left(V \otimes D_{g^{-1}}\right)_{g}, g \in G
$$


is $R$-balanced. Indeed,

$$
\begin{aligned}
(v \diamond r, d) & \mapsto\left(1_{g^{-1}} \delta_{g^{-1}}\right) \diamond(v \diamond r) \otimes \alpha_{g^{-1}}(d) \\
& =\left(\left(1_{g^{-1}} \delta_{g^{-1}}\right)\left(r \delta_{1}\right)\right) \diamond v \otimes \alpha_{g^{-1}}(d) \\
& =\alpha_{g^{-1}}\left(r 1_{g}\right) \delta_{g^{-1}} \diamond v \otimes \alpha_{g^{-1}}(d) \\
& \left.=\left(\left(\alpha_{g^{-1}}\left(r 1_{g}\right) \delta_{1}\right)\left(1_{g^{-1}} \delta_{g^{-1}}\right)\right) \diamond v\right) \otimes \alpha_{g^{-1}}(d) \\
& =\alpha_{g^{-1}}\left(r 1_{g}\right) \delta_{1} \diamond\left(1_{g^{-1}} \delta_{g^{-1}} \diamond v\right) \otimes \alpha_{g^{-1}}(d) \\
& =\left(1_{g^{-1}} \delta_{g^{-1}} \diamond v\right) \diamond\left(\alpha_{g^{-1}}\left(r 1_{g}\right) \delta_{1}\right) \otimes \alpha_{g^{-1}}(d) \\
& =\left(1_{g^{-1}} \delta_{g^{-1}} \diamond v\right) \otimes \alpha_{g^{-1}}(r d),
\end{aligned}
$$

and hence we obtain a well defined map $\psi_{g}: V \otimes D_{g} \rightarrow\left(V \otimes D_{g^{-1}}\right)_{g}$ such that

$$
\psi_{g}(v \otimes d)=\left(1_{g^{-1}} \delta_{g^{-1}}\right) \diamond v \otimes \alpha_{g^{-1}}(d), \text { for all } v \in V, d \in D_{g}
$$

Now given $r \in R$, we have as above

$$
\begin{aligned}
\psi_{g}(r \diamond(v \otimes d)) & =\psi_{g}((r \diamond v) \otimes d) \\
& =\left[\left(1_{g^{-1}} \delta_{g^{-1}}\right) \diamond(r \diamond v)\right] \otimes \alpha_{g^{-1}}(d) \\
& =\left[\left(\alpha_{g^{-1}}\left(r 1_{g}\right) \delta_{g^{-1}}\right) \diamond v\right] \otimes \alpha_{g^{-1}}(d) \\
& =\left[\left(\alpha_{g^{-1}}\left(r 1_{g}\right) \delta_{1}\right) \diamond\left(\left(1_{g^{-1}} \delta_{g^{-1}}\right) \diamond v\right)\right] \otimes \alpha_{g^{-1}}(d) \\
& =\alpha_{g^{-1}}\left(r 1_{g}\right) \diamond \psi_{g}(v \otimes d)
\end{aligned}
$$

and $\psi_{g}$ is $R$-linear. Analogously the map $\lambda_{g}:\left(V \otimes D_{g^{-1}}\right)_{g} \rightarrow V \otimes D_{g}$, satisfying $\lambda_{g}(v \otimes d)=$ $\left(\omega_{g, g^{-1}} \delta_{g}\right) \diamond v \otimes_{R} \alpha_{g}(d)$, for all $v \in V, d \in D_{g^{-1}}$ is well defined and using that $\alpha_{g^{-1}}\left(\omega_{g, g^{-1}}\right)=$ $\omega_{g^{-1}, g}$ we obtain

$$
\left(\psi_{g} \circ \lambda_{g}\right)(v \otimes d)=\left(\left(1_{g^{-1}} \delta_{g^{-1}}\right)\left(\omega_{g, g^{-1}} \delta_{g}\right)\right) \diamond v \otimes d=\left(1_{g^{-1}} \delta_{1} \diamond v\right) \otimes d=v \otimes 1_{g^{-1}} d=v \otimes d,
$$

where the last equality follows because $d \in D_{g^{-1}}$. Hence $\psi_{g} \circ \lambda_{g}=\operatorname{id}_{\left(V \otimes D_{g^{-1}}\right)_{g}}$, and analogously $\lambda_{g} \circ \psi_{g}=\mathrm{id}_{V \otimes D_{g}}$. Therefore $\psi_{g}$ is a $R$-module isomorphism for which $\psi_{g}^{-1}=$ $\lambda_{g}$ and, consequently, $[V] \in \mathbf{P i c S}(R)^{\alpha^{*}}$. Finally, for $g, h \in G, v \in V$ and $d \in D_{g} D_{g h}$ we see that

$$
\begin{aligned}
\psi_{(g h)^{-1}} \psi_{h^{-1}}^{-1} \psi_{g^{-1}}^{-1}(v \otimes d) & =\left[\left(1_{g h} \delta_{g h}\right)\left(\omega_{h^{-1}, h} \delta_{h^{-1}}\right)\left(\omega_{g^{-1}, g} \delta_{g^{-1}}\right)\right] \diamond v \otimes_{R} d \\
& =\left[\left(\alpha_{g h}\left(\omega_{h^{-1}, h} 1_{(g h)^{-1}}\right) \omega_{g h, h^{-1}}^{-1} \delta_{g}\right)\left(\omega_{g^{-1}, g} \delta_{g^{-1}}\right)\right] \diamond v \otimes d \\
& \stackrel{(v)}{=}\left[\left(\omega_{g, h} \delta_{g}\right)\left(\omega_{g^{-1}, g} \delta_{g^{-1}}\right) \diamond v\right] \otimes d \\
& =\left[\left(\omega_{g, h} \alpha_{g}\left(\omega_{g^{-1}, g}\right) \omega_{g, g^{-1}}^{-1} \delta_{1}\right) \diamond v\right] \otimes d \\
& \stackrel{(1)}{=}\left[\left(\omega_{g, h} \delta_{1}\right) \diamond v\right] \otimes d .
\end{aligned}
$$

This yields that $\psi_{3}([V])=\operatorname{cls}(\omega)$ and $\operatorname{im} \varphi_{3} \supseteq \operatorname{ker} \varphi_{4}$.

\section{The homomorphisms $\varphi_{5}$ And exactness at the Brauer group}

We proceed by recalling the construction of the group homomorphism $\varphi_{5}: B\left(R / R^{\alpha}\right) \rightarrow$ $H^{1}\left(G, \alpha^{*}, \mathbf{P i c S}(R)\right)\left(\right.$ see $\left[17\right.$, Section 5.1] for details). Let $\alpha^{*}=\left(\alpha_{g}^{*}, X_{g}\right)_{g \in G}$ be the partial 
action of $G$ on $\operatorname{PicS}(R)$ given in the third item of Lemma 3.8. Then $B^{1}\left(G, \alpha^{*}, \operatorname{PicS}(R)\right)$ is the group

$$
\left\{f \in C^{1}\left(G, \alpha^{*}, \mathbf{P i c S}(R)\right) \mid f(g)=\alpha_{g}^{*}\left([P]\left[D_{g^{-1}}\right]\right)\left[P^{*}\right], \text { for some }[P] \in \mathbf{P i c}(R)\right\}
$$

and $Z^{1}\left(G, \alpha^{*}, \operatorname{PicS}(R)\right)$ is given by

$$
\left\{f \in C^{1}\left(G, \alpha^{*}, \mathbf{P i c S}(R)\right) \mid f(g h)\left[D_{g}\right]=f(g) \alpha_{g}^{*}\left(f(h)\left[D_{g^{-1}}\right]\right), \forall g, h \in G\right\} .
$$

Let $f \in C^{1}\left(G, \alpha^{*}, \operatorname{PicS}(R)\right), g \in G$ and $\mathfrak{p} \in \operatorname{Spec}(R)$. For simplicity of notation we shall write $f(g)_{\mathfrak{p}}$ for the representative of the class $f(g)$ localized at $\mathfrak{p}$.

Let $[A] \in B\left(R / R^{\alpha}\right)$. By $[1$, Theorem 5.7] we may assume that $A$ contains $R$ as a maximal commutative subalgebra. Define the left $R \otimes_{R^{\alpha}} A^{o p^{-m o d u l e}}{ }_{g}\left(1_{g^{-1}} A\right)=1_{g^{-1}} A$ via

$$
\left(r \otimes_{R^{\alpha}} a\right) \bullet a_{g}=\alpha_{g^{-1}}\left(r 1_{g}\right) a_{g} a .
$$

It follows from (14) that $g_{g}\left(1_{g^{-1}} A\right)$ is an object in the category $R \otimes_{R^{\alpha}} A^{o p}$ Mod and there is a unique central $D_{g}$-module $M^{(g)}$ such that

$$
{ }_{g}\left(1_{g^{-1}} A\right) \cong M^{(g)} \otimes A, \text { as }\left(R \otimes_{R^{\alpha}} A^{o p}\right) \text {-modules, }
$$

where $M^{(g)}$ is considered as an $R$-module via the map $r \mapsto r 1_{g}$, and $M^{(g)} \otimes A$ is an $\left(R \otimes_{R^{\alpha}} A^{o p}\right)$-module by means of

$$
\left(r \otimes_{R^{\alpha}} a\right)(x \otimes b)=r x \otimes b a,
$$

$r \in R, a \in A^{o p}, x \in M^{(g)}, b \in A$. Set

$$
f_{A}: G \ni g \mapsto\left[M^{(g)}\right] \in \mathbf{P i c S}(R) .
$$

Proposition 5.1. [17, Theorem 5.9] The map

$$
\varphi_{5}: B\left(R / R^{\alpha}\right) \ni[A] \mapsto \operatorname{cls}\left(f_{A}\right) \in H^{1}\left(G, \alpha^{*}, \operatorname{PicS}(R)\right)
$$

is a group homomorphism.

Remark 5.2. In the proof of Proposition 5.1 the following was obtained. Let $A_{1}, A_{2}$ be two $R^{\alpha}$-Azumaya algebras containing $R$ as a maximal commutative subalgebra, and let $M_{1}^{(g)}, M_{2}^{(g)}$ be two $R$-modules such that there are isomorphism ${ }_{g}\left(1_{g^{-1}} A\right) \cong M_{1}^{(g)} \otimes A_{1}$, ${ }_{g}\left(1_{g^{-1}} A_{2}\right) \cong M_{2}^{(g)} \otimes A_{2}$ as $R \otimes_{R^{\alpha}} A_{1}^{o p}$-modules and $R \otimes_{R^{\alpha}} A_{2}^{o p}$-modules respectively. If $B=$ $A_{1} \otimes_{R^{\alpha}} A_{2}$ and $e$ is the separability idempotent of the ring extension $R / R^{\alpha}$, then eBe is an $R^{\alpha}$-Azumaya algebra containing $R$ as a maximal commutative subalgebra and there is an $R \otimes_{R^{\alpha}}(e B e)^{o p}$-module isomorphism

$$
{ }_{g}\left(1_{g^{-1}} e B e\right) \cong\left(M_{1}^{(g)} \otimes M_{2}^{(g)}\right) \otimes e B e .
$$

Moreover, $[B]=[e B e] \in B\left(R / R^{\alpha}\right)$.

By [17, Theorem 3.8] we have that $\left[M^{(g)}\right] \in \operatorname{Pic}\left(D_{g}\right)$, for all $g \in G$. For further reference, we shall construct $M^{(g)}$ explicitly.

We have the following. 
Lemma 5.3. Let $A$ be an Azumaya $R^{\alpha}$-algebra containing $R$ as a maximal commutative subalgebra. Write

$$
\left.C_{g}=C_{g^{(1} g^{-1}} A\right)(R)=\left\{c \in 1_{g^{-1}} A \mid \alpha_{g^{-1}}\left(r 1_{g}\right) c=c r, r \in R\right\},
$$

for each $g \in G$. Then there is a left $R \otimes_{R^{\alpha}} A^{\text {op }}$-module isomorphism ${ }_{g}\left(1_{g^{-1}} A\right) \cong C_{g} \otimes_{R} A$.

Proof. Since $R / R^{\alpha}$ is a partial Galois extension then by [13, Theorem 4.2] the extension $R / R^{\alpha}$ is separable, moreover since $A$ contains $R$ as a maximal commutative subalgebra we obtain from [22, Theorem 7.4.2] that $A$ is a faithfully projective $R$-module and by $[22$, Excercise 7.4 .7 p. 258] that the functor

$$
-\otimes_{R} A: \operatorname{Mod}_{R} \rightarrow_{R \otimes_{R^{\alpha}} A^{o p} \operatorname{Mod}}
$$

determines an equivalence of categories, whose inverse is given by the functor

$$
(-)^{R}:{ }_{\left(R \otimes_{R^{\alpha}} A^{o p}\right)} \operatorname{Mod} \rightarrow \operatorname{Mod}_{R},
$$

where

$$
N^{R}=\left\{x \in N \mid\left(r \otimes_{R^{\alpha}} 1\right) x=\left(1 \otimes_{R^{\alpha}} r\right) x, \forall r \in R\right\},
$$

for each $N$ in ${ }_{\left(R \otimes_{R^{\alpha}} A^{o p}\right)}$ Mod. In particular, for any left $R \otimes_{R^{\alpha}} A^{o p}$-module $N$ there is a left $R \otimes_{R^{\alpha}} A^{o p}$-module isomorphism $N \cong N^{R} \otimes_{R} A$. Then taking $N={ }_{g^{-1}}\left(1_{g} A\right)$, we have $N^{R}=C_{g}$, and the desired isomorphism follows.

Remark 5.4. It follows from Lemma 5.3 that for any $[A] \in B\left(R / R^{\alpha}\right)$ one has that $\varphi_{5}([A])=\operatorname{cls}(f)$, where $f(g)=\left[C_{g}\right]$ for any $g \in G$.

Now we give:

Lemma 5.5. Let $R / R^{\alpha}$ be a partial Galois extension. Then for an arbitrary $[J] \in$ $\operatorname{Pic}(R)$ there is an $R \otimes_{R^{\alpha}}\left(\operatorname{End}_{R^{\alpha}}(J)\right)^{o p}$-module isomorphism

$$
{ }_{g}\left(1_{g^{-1}} \operatorname{End}_{R^{\alpha}}(J)\right) \cong{ }_{g}\left(1_{g^{-1}} J^{*}\right) \otimes J \otimes\left(\operatorname{End}_{R^{\alpha}}(J)\right),
$$

where $J^{*}=\operatorname{Hom}_{R}(J, R)$, and the $R$-module structure of $g_{g}\left(1_{g^{-1}} J^{*}\right)$ is given by (5).

Proof. Let $g \in G$, since $\left[J^{*}\right]$ is the inverse of $[J]$ in $\mathbf{P i c}(R) \subseteq \mathbf{P i c S}(R)$ and $\left[D_{g^{-1}}\right]$ is an idempotent in $\mathbf{P i c S}(R)$ there are isomorphisms of $R$-modules

$$
1_{g^{-1}} J^{*} \cong D_{g^{-1}} \otimes J^{*} \cong D_{g^{-1}}^{*} \otimes J^{*} \cong\left(D_{g^{-1}} \otimes J\right)^{*} \cong\left(1_{g^{-1}} J\right)^{*}
$$

and consequently, there is an $R$-module isomorphism $g\left(1_{g^{-1}} J^{*}\right) \stackrel{(6)}{\cong}\left(g_{g}\left(1_{g^{-1}} J\right)\right)^{*}$, where

$$
\left({ }_{g}\left(1_{g^{-1}} J\right)\right)^{*}=\left\{\eta: 1_{g^{-1}} J \rightarrow R \mid \eta\left(\alpha_{g^{-1}}\left(r 1_{g}\right) x\right)=r \eta(x), \forall r \in R, x \in 1_{g^{-1}} J\right\} .
$$

Now set $C_{g}=\left\{\xi \in 1_{g} \operatorname{End}_{R^{\alpha}}(J) \mid \alpha_{g}\left(r 1_{g^{-1}}\right) \xi(p)=\xi(r p), \forall p \in J, r \in R\right\}$.

Since $\operatorname{End}_{R^{\alpha}}(J)$ is an Azumaya $R^{\alpha}$-algebra containing $R$ as a maximal commutative $R^{\alpha}$-algebra, then by Lemma 5.3 it is enough to show that there is an $R$-module isomorphism

$$
\left({ }_{g}\left(1_{g^{-1}} J\right)\right)^{*} \otimes J \cong C_{g}
$$

For this purpose notice that for each $\eta \in\left({ }_{g}\left(1_{g^{-1}} J\right)\right)^{*}$ and $x \in J$ the map

$$
\eta_{x}: J \ni p \mapsto \eta\left(1_{g^{-1}} p\right) x \in J
$$


belongs to $\operatorname{End}_{R^{\alpha}}(J)$. For, given $r \in R^{\alpha}$ and $p \in J$ we have that

$$
\eta_{x}(r p)=\eta\left(r 1_{g^{-1}} p\right) x=\eta\left(\alpha_{g^{-1}}\left(r 1_{g}\right) p\right) x=r \eta\left(1_{g^{-1}} p\right) x=r \eta_{x}(p) .
$$

Furthermore, $1_{g} \eta_{x} \in C_{g}$, because,

$$
\begin{aligned}
\alpha_{g}\left(r 1_{g^{-1}}\right)\left(1_{g} \eta_{x}(p)\right) & =\alpha_{g}\left(r 1_{g^{-1}}\right) \eta\left(1_{g^{-1}} p\right) x \\
& \stackrel{(18)}{=} \eta\left[\alpha_{g^{-1}}\left(\alpha_{g}\left(r 1_{g^{-1}}\right)\right) p\right] x \\
& =\eta\left(1_{g^{-1}} r p\right) x \\
& =\left(1_{g} \eta_{x}\right)(r p),
\end{aligned}
$$

for each $r \in R$ and $p \in J$. Since $\eta_{r x}=r \eta_{x}$, for all $r \in R$ and $x \in J$, we have an $R$-linear map $\vartheta:\left({ }_{g}\left(1_{g^{-1}} J\right)\right)^{*} \otimes J \ni \eta \otimes x \mapsto 1_{g} \eta_{x} \in C_{g}$, and we shall prove that $\vartheta$ is bijective.

$\vartheta$ is onto. Consider a dual basis $\left\{x_{i}, \eta_{i}\right\}$ for the projective $R$-module $g\left(1_{g^{-1}} J\right)$, take an arbitrary $\xi \in C_{g}$. Notice first that for any $\eta \in\left(g_{(}\left(1_{g^{-1}} J\right)\right)^{*}$ the map

$$
\tilde{\eta}: 1_{g^{-1}} J \ni x \mapsto \alpha_{g^{-1}}\left(1_{g} \eta\left(1_{g^{-1}} \xi(x)\right)\right) \in R
$$

is an element of $\left({ }_{g}\left(1_{g^{-1}} J\right)\right)^{*}$ because for any $r \in R$ and $x \in 1_{g} J$ we have

$$
\begin{aligned}
\left.\tilde{\eta}\left(\alpha_{g^{-1}}\left(r 1_{g}\right) x\right)\right) & =\alpha_{g^{-1}}\left\{1_{g} \eta\left[1_{g^{-1}} \xi\left(\alpha_{g^{-1}}\left(r 1_{g}\right) x\right)\right]\right\} \\
& \stackrel{\xi \in C_{g}}{=} \alpha_{g^{-1}}\left[\eta\left(r 1_{g^{-1}} \xi(x)\right)\right] \\
& \stackrel{(18)}{=} \alpha_{g^{-1}}\left(\alpha_{g}\left(r 1_{g^{-1}}\right) \eta\left(1_{g^{-1}} \xi(x)\right)\right) \\
& =r \alpha_{g^{-1}}\left(1_{g} \eta\left(1_{g^{-1}} \xi(x)\right)\right) \\
& =r \tilde{\eta}(x) .
\end{aligned}
$$

Taking specifically the $\tilde{\eta}_{i}$ and $x \in 1_{g} J$ we have

$$
\tilde{\eta}_{i_{x_{i}}}(x)=\tilde{\eta}_{i}(x) x_{i}=\alpha_{g^{-1}}\left(1_{g} \eta_{i}(\xi(x))\right) x_{i}=\eta_{i}(\xi(x)) \bullet x_{i} .
$$

From this, using the fact that $\left\{x_{i}, \eta_{i}\right\}$ is a dual basis, we conclude that $\sum_{i} \tilde{\eta}_{i_{x_{i}}}(x)=$ $\sum_{i} \eta_{i}(\xi(x)) \bullet x_{i}=\xi(x)$. Thus, $\vartheta\left(\sum_{i} x_{i} \otimes \tilde{\eta}_{i}\right)=1_{g} \sum_{i}\left(\tilde{\eta}_{i}\right)_{x_{i}}=1_{g} \xi=\xi$, showing that $\vartheta$ is surjective.

$\vartheta$ is injective. Let $c=\sum_{j} v_{j} \otimes z_{j} \in \operatorname{ker} \vartheta$. Then $0=1_{g} \sum_{j}\left(v_{j}\right)_{z_{j}}$. Consequently, for any $p \in J$ we have

$$
0=1_{g} \sum_{j}\left(v_{j}\right)_{z_{j}}(p)=\sum_{j} 1_{g} v_{j}\left(1_{g^{-1}} p\right) z_{j}=\sum_{j} v_{j}\left(1_{g^{-1}} p\right) z_{j}=\sum_{j}\left(v_{j}\right)_{z_{j}}(p),
$$

and $\sum_{j}\left(v_{j}\right)_{z_{j}}=0$. Let $\left\{p_{l}, \xi_{l}\right\}$ be a dual $R$-basis for $J$. Then

$$
c=\sum_{j} z_{j} \otimes v_{j}=\sum_{j, l} \xi_{l}\left(z_{j}\right) p_{l} \otimes v_{j}=\sum_{l} p_{l} \otimes \sum_{j} \xi_{l}\left(z_{j}\right) v_{j} .
$$


Since for any $x \in 1_{g^{-1}} J$ we have

$$
\left(\sum_{j} \xi_{l}\left(z_{j}\right) v_{j}\right)(x)=\xi_{l}\left(\sum_{j} z_{j} v_{j}(x)\right)=\xi_{l}\left(\sum_{j}\left(v_{j}\right)_{z_{j}}(x)\right)=0,
$$

and we conclude that $c=0$ thus $\vartheta$ is injective.

Lemma 5.6. Let $A$ be an Azumaya $R^{\alpha}$-algebra containing $R$ as a maximal commutative subalgebra. If there exists a $R \otimes_{R^{\alpha}} A^{o p}$-module isomorphism ${ }_{g}\left(1_{g^{-1}} A\right) \simeq 1_{g} A$, then $A=$ $R \star_{\alpha, \omega} G$, for some $\omega \in H^{2}(G, \alpha, R)$.

Proof. Let $\theta_{g}:{ }_{g}\left(1_{g^{-1}} A\right) \rightarrow 1_{g} A$ be an $R \otimes_{R^{\alpha}} A^{o p}$-module isomorphism. Write $\theta_{g}\left(1_{g^{-1}}\right)=u_{g}$. We shall construct an $R^{\alpha}$-algebra $\mathcal{B}$ which is isomorphic to a crossed product by a twisting of $\alpha$. To this we establish first some properties of the family $\left\{u_{g}\right\}_{g \in G}$. For $r \otimes_{R^{\alpha}} a \in R \otimes_{R^{\alpha}} A^{o p}$, we have

$$
\left(r \otimes_{R^{\alpha}} a\right) \bullet 1_{g^{-1}}=\alpha_{g^{-1}}\left(r 1_{g}\right) a=\left(1 \otimes_{R^{\alpha}} \alpha_{g^{-1}}\left(r 1_{g}\right) a\right) \bullet\left(1_{g^{-1}}\right) .
$$

Applying $\theta_{g}$ we get

$$
\left(r \otimes_{R^{\alpha}} a\right) u_{g}=\left(1_{g} \otimes_{R^{\alpha}} \alpha_{g^{-1}}\left(r 1_{g}\right) a\right) u_{g},
$$

or, $r u_{g} a=u_{g} \alpha_{g^{-1}}\left(r 1_{g}\right) a$ In particular, letting $a=1$ we get

$$
r u_{g}=u_{g} \alpha_{g^{-1}}\left(r 1_{g}\right), \text { for any } r \in R \text { and } g \in G .
$$

It follows from (19) that

$$
u_{g}=u_{g} 1_{g^{-1}} \quad \text { for all } g \in G .
$$

Now, take $a_{g} \in 1_{g^{-1}} A$, such that $\theta_{g}\left(a_{g}\right)=1_{g}$. Then, $1_{g}=\theta_{g}\left(\left(1_{g} \otimes a_{g}\right) \bullet\left(1_{g^{-1}}\right)\right)=$ $\left(1_{g} \otimes a_{g}\right) \theta_{g}\left(1_{g^{-1}}\right)=u_{g} a_{g}$, and we conclude that

$$
1_{g}=u_{g} a_{g}, \text { for all } g \in G \text {. }
$$

Moreover, $\theta_{g}\left(a_{g} u_{g}\right)=\left(1_{g} \otimes u_{g}\right) \theta_{g}\left(a_{g}\right)=u_{g}$, and since $\theta_{g}$ is injective we get

$$
1_{g^{-1}}=a_{g} u_{g}
$$

and follows from (19) that for each $r \in R$ we have $a_{g} r u_{g}=a_{g} u_{g} \alpha_{g^{-1}}\left(r 1_{g}\right) \stackrel{(22)}{=} \alpha_{g^{-1}}\left(r 1_{g}\right)$, which gives

$$
a_{g} r 1_{g} \stackrel{(21)}{=}\left(a_{g} r u_{g}\right) a_{g}=\alpha_{g^{-1}}\left(r 1_{g}\right) a_{g},
$$

since $a_{g} \in 1_{g^{-1}} A$ we get

$$
a_{g} r=\alpha_{g^{-1}}\left(r 1_{g}\right) a_{g}
$$


for all $g \in G$. Let $w_{g, h}=u_{g} u_{h} a_{g h}$, for all $g, h \in R$. Notice that $w_{g, h} \in R$, because for each $r \in R$

$$
\begin{aligned}
r w_{g, h} & =r u_{g} u_{h} a_{g h} \\
& \stackrel{(19)}{=} u_{g} u_{h} \alpha_{h^{-1}}\left(\alpha_{g^{-1}}\left(r 1_{g}\right) 1_{h}\right) a_{g h} \\
& =u_{g} u_{h} \alpha_{(g h)^{-1}}\left(r 1_{g h}\right) 1_{h^{-1}} a_{g h} \\
& \stackrel{(20)}{=} u_{g} u_{h} \alpha_{(g h)^{-1}}\left(r 1_{g h}\right) a_{g h} \\
& \stackrel{(23)}{=} u_{g} u_{h} a_{g h} r .
\end{aligned}
$$

Now we check that $w_{g, h} \in D_{g} D_{g h}$, indeed

$$
\begin{aligned}
w_{g, h} 1_{g} 1_{g h} & =1_{g} u_{g} u_{h} a_{g h} 1_{g h} \\
& =u_{g} u_{h} a_{g h} 1_{g h} \\
& \stackrel{(23)}{=} u_{g} u_{h} 1_{(g h)^{-1}} a_{g h} \\
& \stackrel{(20)}{=} u_{g} u_{h} 1_{h^{-1}} 1_{(g h)^{-1}} a_{g h} \\
& =u_{g} u_{h} \alpha_{h^{-1}}\left(1_{g^{-1}} 1_{h}\right) a_{g h} \\
& \stackrel{(19)}{=}\left(u_{g} 1_{g^{-1}}\right) u_{h} a_{g h} \\
& \stackrel{(20)}{=} u_{g} u_{h} a_{g h} .
\end{aligned}
$$

In an analogous way one can show that $r_{g, h}=u_{g h} a_{h} a_{g} \in D_{g} D_{g h}$. Thus, for any $g, h \in G$ we see that

$$
\begin{aligned}
w_{g, h} r_{g, h} & =u_{g} u_{h} a_{g h} u_{g h} a_{h} a_{g} \\
& =u_{g} u_{h} 1_{(g h)^{-1}} a_{h} a_{g} \\
& =u_{g} u_{h} a_{h} a_{g} \\
& =u_{g} 1_{h} a_{g} \\
& =u_{g} 1_{h} 1_{g^{-1}} a_{g} \\
& =u_{g} \alpha_{g^{-1}}\left(1_{g h} 1_{g}\right) a_{g} \\
& =1_{g h} 1_{g} u_{g} a_{g} . \\
& =1_{g h} 1_{g} .
\end{aligned}
$$

Hence to prove that the family $w=\left\{w_{g, h}\right\}_{(g, h) \in G \times G}$ is a twisting for $\alpha$ we need to check equality (v). For any $g, h, l \in G$ we have

$$
w_{g, h} w_{g h, l}=u_{g} u_{h} a_{g h} u_{g h} u_{l} a_{g h l} \stackrel{(22)}{=} u_{g} u_{h} 1_{(g h)^{-1}} u_{l} a_{g h l}=u_{g} u_{h} u_{l} a_{g h l},
$$

and

$$
\alpha_{g}\left(w_{h, l} 1_{g^{-1}}\right) w_{g, h l}=\alpha_{g}\left(r_{h, l} 1_{g^{-1}}\right) u_{g} u_{h l} a_{g h l} \stackrel{(19)}{=} u_{g} u_{h} u_{l} a_{h l} u_{h l} a_{g h l} \stackrel{(22)}{=} u_{g} u_{h} u_{l} a_{g h l} .
$$


Set $\mathcal{B}=\sum_{g \in G} \mathcal{B}_{g} \subseteq A$, where $\mathcal{B}_{g}=D_{g} u_{g}$, for any $g \in G$. Notice that by (19) we get $\alpha_{g}\left(r 1_{g^{-1}}\right) u_{g}=u_{g} r$, for all $r \in R$ and $g \in G$, moreover $w_{g, h} u_{g, h}=u_{g} u_{h} 1_{(g h)^{-1}}=u_{g} u_{h}$. Then by (2) the map

$$
\digamma: R \star_{\alpha, w} G \ni \sum_{g \in G} a_{g} \delta_{g} \rightarrow \sum_{g \in G} a_{g} u_{g} \in \mathcal{B}
$$

is an $R^{\alpha}$-algebra epimorphism. Since $R \star_{\alpha, w} G$ is an Azumaya $R^{\alpha}$-algebra, [7, Corollary 2.3.7] implies ker $\digamma=\left(\operatorname{ker} \digamma \cap R^{\alpha}\right)\left(R \star_{\alpha, w} G\right)$. Moreover, the fact that $u_{1} \in \mathcal{U}(R)$, yields that $\digamma$ restricted to $R^{\alpha}$ is injective, and, consequently, $\digamma$ is a ring monomorphism. This yields that $\mathcal{B}=\bigoplus_{g \in G} \mathcal{B}_{g} \cong R \star_{\alpha, \mathfrak{r}} G$ as $R^{\alpha}$-algebras, and we may assume that $R \star_{\alpha, w} G \subseteq A$.

We check that $C_{A}\left(R \star_{\alpha, w} G\right)=R^{\alpha}$. From $R \subseteq R \star_{\alpha, \mathfrak{r}} G$, we obtain that

$$
R^{\alpha} \subseteq C_{A}\left(R \star_{\alpha, w} G\right) \subseteq C_{A}(R)=R
$$

Take $x \in C_{A}\left(R \star_{\alpha, w} G\right)$, then $x \in R$ and for all $g \in G$ we have $x\left(1_{g} \delta_{g}\right)=\left(1_{g} \delta_{g}\right) x$, or equivalently $x 1_{g}=\alpha_{g}\left(x 1_{g^{-1}}\right)$, which gives $x \in R^{\alpha}$, showing that $C_{A}\left(R \star_{\alpha, w} G\right)=R^{\alpha}$.

Since $R \star_{\alpha, w} G$ is an Azumaya $R^{\alpha}$-subalgebra of $A$, the double centralizer Theorem (see [7, Theorem 2.4.3]) implies $R \star_{\alpha, w} G=C_{A}\left(C_{A}\left(R \star_{\alpha, w} G\right)\right)=C_{A}\left(R^{\alpha}\right)=A$.

Theorem 5.7. The sequence $H^{2}(G, \alpha, R) \stackrel{\varphi_{4}}{\rightarrow} B\left(R / R^{\alpha}\right) \stackrel{\varphi_{5}}{\rightarrow} H^{1}\left(G, \alpha^{*}, \mathbf{P i c S}(R)\right)$ is exact.

Proof. Let $\operatorname{cls}(\omega) \in H^{2}(G, \alpha, R)$ and $\varphi_{5}\left(\left[R \star_{\alpha, \omega} G\right]\right)=\operatorname{cls}(f)$. We shall check that $f \in B^{1}\left(G, \alpha^{*}, \mathbf{P i c S}(R)\right)$ by proving that $f(g)=\left[D_{g}\right]$, for any $g \in G$. For this we need to show that $g_{g}\left(D_{g^{-1}} \otimes R \star_{\alpha, \omega} G\right)$ and $D_{g} \otimes R \star_{\alpha, \omega} G$ are isomorphic as $D_{g} \otimes_{R^{\alpha}}\left(R \star_{\alpha, \omega} G\right)^{o p_{-}}$ modules.

Notice that the map

$$
D_{g^{-1}} \times R \star_{\alpha, \omega} G \ni\left(d, \sum_{h \in G} a_{h} \delta_{h}\right) \mapsto 1_{g} \otimes \sum_{h \in G} \alpha_{g}\left(d a_{h}\right) \omega_{g, h} \delta_{g h} \in D_{g} \otimes R \star_{\alpha, \omega} G
$$

is $R$-balanced, so that it induces a well defined map

$$
\nu_{g}:{ }_{g}\left(D_{g^{-1}} \otimes R \star_{\alpha, \omega} G\right) \ni d \otimes \sum_{h \in G} a_{h} \delta_{h} \mapsto 1_{g} \otimes \sum_{h \in G} \alpha_{g}\left(d a_{h}\right) \omega_{g, h} \delta_{g h} \in D_{g} \otimes R \star_{\alpha, \omega} G .
$$



have

Now for $d_{g} \otimes_{R^{\alpha}} a_{l} \delta_{l} \in D_{g} \otimes_{R^{\alpha}}\left(R \star_{\alpha, \omega} G\right)^{o p}$ and $d \otimes \sum_{h \in G} a_{h} \delta_{h} \in{ }_{g}\left(D_{g^{-1}} \otimes R \star_{\alpha, \omega} G\right)$, we

$$
\begin{aligned}
& \left(d_{g} \otimes a_{l} \delta_{l}\right) \bullet\left(d \otimes \sum_{h \in G} a_{h} \delta_{h}\right)=\alpha_{g^{-1}}\left(d_{g}\right) d \otimes \sum_{h \in G} a_{h} \alpha_{h}\left(a_{l} 1_{h^{-1}}\right) \omega_{h, l} \delta_{h l} \\
& \stackrel{\nu_{g}}{\mapsto} 1_{g} \otimes \sum_{h \in G} d_{g} \alpha_{g}\left(d a_{h} \alpha_{h}\left(a_{l} 1_{h^{-1}}\right) \omega_{h, l}\right) \omega_{g, h l} \delta_{g h l} \\
& \stackrel{(4)}{=} d_{g} \otimes \sum_{h \in G} \alpha_{g}\left(d a_{h}\right) \alpha_{g h}\left(a_{l} 1_{(g h)^{-1}}\right) \alpha_{g}\left(\omega_{h, l} 1_{g^{-1}}\right) \omega_{g, h l} \delta_{g h l} \\
& =d_{g} \otimes \sum_{h \in G} \alpha_{g}\left(d a_{h}\right) \omega_{g, h} \alpha_{g h}\left(a_{l} 1_{(g h)^{-1}}\right) \omega_{g h, l} \delta_{g h l} \\
& =d_{g}\left(1_{g} \otimes \sum_{h \in G} \alpha_{g}\left(d a_{h}\right) \omega_{g, h} \delta_{g h}\right)\left(a_{l} \delta_{l}\right) \\
& =\left(d_{g} \otimes a_{l} \delta_{l}\right) \nu_{g}\left(d \otimes \sum_{h \in G} a_{h} \delta_{h}\right)
\end{aligned}
$$

and $\nu_{g}$ is $D_{g} \otimes_{R^{\alpha}}\left(R \star_{\alpha, \omega} G\right)^{o p}$-linear. To prove that $\nu_{g}$ is injective consider the map

$$
D_{g} \otimes R \star_{\alpha, \omega} G \ni d^{\prime} \otimes \sum_{h \in G} a_{h} \delta_{h} \stackrel{\lambda_{g}}{\mapsto} 1_{g^{-1}} \otimes \sum_{h \in G} \alpha_{g^{-1}}\left(d^{\prime} a_{h} \omega_{g, h}^{-1}\right) \delta_{g^{-1} h} \in{ }_{g}\left(D_{g^{-1}} \otimes R \star_{\alpha, \omega} G\right) .
$$

It is easy to see that $\lambda_{g} \nu_{g}=\operatorname{id}_{g}\left(D_{g^{-1}} \otimes R \star_{\alpha, \omega} G\right)$, and $\nu_{g}$ is injective.

For the surjectivity, consider $d_{g} \otimes \sum_{h \in G} a_{g h} \delta_{g h}=1_{g} \otimes \sum_{h \in G} d_{g} a_{g h} \delta_{g h} \in D_{g} \otimes R \star_{\alpha, \omega} G$. Then,

$$
\nu_{g}\left(\alpha_{g^{-1}}\left(d_{g}\right) \otimes \sum_{h \in G} \alpha_{g^{-1}}\left(a_{g h} \omega_{g, h}^{-1}\right) \delta_{h}\right)=1_{g} \otimes \sum_{h \in G} d_{g} a_{g h} \omega_{g, h}^{-1} \omega_{g, h} \delta_{g h}=d_{g} \otimes \sum_{h \in G} a_{g h} \delta_{g h} .
$$

and $\nu_{g}$ is surjective. Using (15) we get $f(g)=\left[D_{g}\right]$, which implies $\varphi\left(\left[R \star_{\alpha, \omega} G\right]\right)=\operatorname{cls}(1)$ in $H^{1}\left(G, \alpha^{*}, \mathbf{P i c S}(R)\right)$ and $\operatorname{im} \varphi_{4} \subseteq \operatorname{ker} \varphi_{5}$.

Now we prove $\operatorname{im} \varphi_{4} \supseteq \operatorname{ker} \varphi_{5}$. Let $[A] \in \operatorname{ker} \varphi_{5}$ with $R$ being a maximal commutative subalgebra of $A$. Then, $f=f_{A} \in B^{1}\left(G, \alpha^{*}, \mathbf{P i c} S(R)\right)$, where $f_{A}$ is given by (16), and there exists $[P] \in \mathbf{P i c}(R)$ such that $M^{(g)} \cong\left(1_{g^{-1}} P\right)_{g} \otimes P^{*}$ as $R$-modules. By the construction of $\varphi_{5}$, the latter implies that there is an $R \otimes_{R^{\alpha}} A^{o p}$-module isomorphism

$$
{ }_{g}\left(1_{g^{-1}} A\right) \cong_{g}\left(1_{g^{-1}} P\right) \otimes P^{*} \otimes A .
$$

Since $D_{g}$ is a direct summand of $R$, the map $\operatorname{Pic}(R) \ni[Q] \rightarrow\left[D_{g} \otimes Q\right] \in \operatorname{Pic}\left(D_{g}\right)$ is a group epimorphism, for each $g \in G$. Consequently, $\left[1_{g^{-1}} P\right]=\left[D_{g^{-1}} \otimes P\right] \in \mathbf{P i c}\left(D_{g^{-1}}\right)$ and $\left[{ }_{g}\left(1_{g^{-1}} P\right)\right] \in \mathbf{P i c}\left(D_{g}\right)$ by the first item of Lemma 3.8. Using again the above epimorphism, we see that there exists $[J] \in \mathbf{P i c}(R)$ and a $D_{g}$-module isomorphism ${ }_{g}\left(1_{g^{-1}} P\right) \cong D_{g} \otimes J^{*} \cong 1_{g} J^{*}$.

Applying $\alpha_{g}{ }^{*}$ to both parts of the equality we obtain an $R$-module isomorphism $1_{g^{-1}} P \cong g^{-1}\left(1_{g} J^{*}\right)$, Moreover, $\left(1_{g^{-1}} P^{*}\right)_{g} \cong 1_{g} J$, as $R$-modules, in view of $(6)$. By 
Lemma 5.5 there exists a $R \otimes_{R^{\alpha}}\left(\operatorname{End}_{R^{\alpha}}(J)\right)^{o p}$-module isomorphism

$$
{ }_{g}\left(1_{g^{-1}} \operatorname{End}_{R^{\alpha}}(J)\right) \cong g^{-1}\left(1_{g} J^{*}\right) \otimes J \otimes \operatorname{End}_{R^{\alpha}}(J) .
$$

Since $g_{g}\left(1_{g^{-1}} \operatorname{End}_{R^{\alpha}}(J)\right)$ is a left unital $D_{g}$ module, tensoring by $D_{g}$ we obtain

$$
\begin{aligned}
g_{g}\left(1_{g^{-1}} \operatorname{End}_{R^{\alpha}}(J)\right) & \cong D_{g} \otimes g^{-1}\left(1_{g} J^{*}\right) \otimes J \otimes \operatorname{End}_{R^{\alpha}}(J) \\
& \cong D_{g} \otimes 1_{g^{-1}} P \otimes J \otimes \operatorname{End}_{R^{\alpha}}(J) \\
& \cong 1_{g^{-1}} P \otimes D_{g} \otimes J \otimes \operatorname{End}_{R^{\alpha}}(J) \\
& \cong 1_{g^{-1}} P \otimes 1_{g} J \otimes \operatorname{End}_{R^{\alpha}}(J) \\
& \cong 1_{g^{-1}} P \otimes g_{g}\left(1_{g^{-1}} P^{*}\right) \otimes \operatorname{End}_{R^{\alpha}}(J),
\end{aligned}
$$

as $R \otimes_{1_{g} R^{\alpha}} \operatorname{End}_{R^{\alpha}}(J)^{o p}$-modules. Let $e$ be an idempotent of separability of $R$ over $R^{\alpha}$. It follows from (17), (24) and the latter isomorphism, that taking $\Delta=A \otimes_{R^{\alpha}} \operatorname{End}_{R^{\alpha}}(J)$ one has as $R \otimes_{R^{\alpha}} \Delta^{o p}$-modules that

$$
\begin{aligned}
g_{g}\left(1_{g^{-1}} e \Delta e\right) & \cong\left\{{ }_{g}\left(1_{g^{-1}} P\right) \otimes P^{*}\right\} \otimes\left\{1_{g^{-1}} P \otimes_{g}\left(1_{g^{-1}} P^{*}\right)\right\} \otimes e \Delta e \\
& \cong\left\{{ }_{g}\left(1_{g^{-1}} P\right) \otimes\left(P^{*} \otimes 1_{g^{-1}} P\right) \otimes_{g}\left(1_{g^{-1}} P^{*}\right)\right\} \otimes e \Delta e \\
& \cong\left\{{ }_{g}\left(1_{g^{-1}} P\right) \otimes D_{g^{-1}} \otimes_{g}\left(1_{g^{-1}} P^{*}\right)\right\} \otimes e \Delta e \\
& \cong\left\{{ }_{g}\left(1_{g^{-1}} P\right) \otimes_{g}\left(1_{g^{-1}} P^{*}\right)\right\} \otimes e \Delta e \\
& \cong{ }_{g}\left(D_{g^{-1}}\right) \otimes e \Delta e \\
& \cong D_{g} \otimes e \Delta e .
\end{aligned}
$$

Since $[e \Delta e]=[\Delta]=[A]$ in $B\left(R^{\alpha}\right)$, replacing $e \Delta e$ by $A$, we may suppose, without loss of generality, that ${ }_{g}\left(1_{g^{-1}} A\right) \cong 1_{g} A$, as $R \otimes_{R^{\alpha}} A^{o p}$-modules. By Lemma 5.6, we have that $A=R \star_{\alpha, \omega} G$, for some $\omega \in H^{2}(G, \alpha, R)$. Then, $\varphi_{4}(\operatorname{cls}(\omega))=\left[R \star_{\alpha, \omega} G\right]=[A]$, and $[A] \in \operatorname{im} \varphi_{4}$.

$$
\text { 6. The homomorphism } \varphi_{6}: H^{1}\left(G, \alpha^{*}, \operatorname{PicS}(R)\right) \rightarrow H^{3}(G, \alpha, R)
$$

We start by recalling from [17] the definition of the $R$ - $R$-bimodule ${ }_{g}\left(D_{g^{-1}}\right)_{I}, g \in G$ : its underlying set is $D_{g^{-1}}$, endowed with the action $*$ given by

$$
r * d=\alpha_{g^{-1}}\left(r 1_{g}\right) d, \text { and } d * r=d r, \text { for any } r \in R, d \in D_{g^{-1}} .
$$

Write for simplicity $g\left(D_{g^{-1}}\right)={ }_{g}\left(D_{g^{-1}}\right)_{I}$. Now we give from [12] the concept of a partial representation.

Definition 6.1. A (unital) partial representation of $G$ into an algebra (or, more generally, a monoid) $S$ is a map $\Phi: G \rightarrow S$ which satisfies the following properties, for all $g, h \in G$,

(i) $\Phi\left(g^{-1}\right) \Phi(g) \Phi(h)=\Phi\left(g^{-1}\right) \Phi(g h)$,

(ii) $\Phi(g) \Phi(h) \Phi\left(h^{-1}\right)=\Phi(g h) \Phi\left(h^{-1}\right)$,

(iii) $\Phi\left(1_{G}\right)=1_{S}$.

We also recall two important for us partial representations of $G$. 
Proposition 6.2. Let $\Phi_{0}: G \ni g \mapsto\left[{ }_{g}\left(D_{g^{-1}}\right)\right] \in \mathbf{P i c S}_{R^{\alpha}}(R)$ and for any cocycle $f \in$ $Z^{1}\left(G, \alpha^{*}, \operatorname{PicS}(R)\right)$, set $\Phi_{f}=f \Phi_{0}: G \rightarrow \operatorname{PicS}_{R^{\alpha}}(R)$, that is $\Phi_{f}(g)=f(g) \Phi_{0}(g)$, for any $g \in G$. Then,

- [17, Proposition 6.2] $\Phi_{0}$ is a partial representation with $\Phi_{0}(g) \Phi_{0}\left(g^{-1}\right)=\left[D_{g}\right]$.

- $\left[17\right.$, Lemma 6.3] $\Phi_{f}$ is a partial representation of $G$, with $\Phi_{f}(g) \Phi_{f}\left(g^{-1}\right)=\left[D_{g}\right]$. Moreover, writing $\Phi_{f}(g)=\left[J_{g}\right]$, we have that $D_{g} \cong \operatorname{End}_{D_{g}}\left(J_{g}\right)$, as $R$ - and $D_{g^{-}}$ algebras, for any $g \in G$.

Remark 6.3. Let $f$ be an element of $Z^{1}\left(G, \alpha^{*}, \operatorname{PicS}(R)\right)$ and $J_{g}=f(g) \otimes_{g}\left(D_{g^{-1}}\right)$. Then

- [17, Remark 6.5] For $x_{g} \in J_{g}$ and $r \in R$ we have.

$$
\alpha_{g}\left(r 1_{g^{-1}}\right) x_{g}=x_{g} r
$$

and if $D_{g}$ is a semi-local ring, then for any $g \in G$, we see that there is $u_{g} \in J_{g}$, a $D_{g}$-basis of $J_{g}$ with $J_{g}=D_{g} u_{g}$, and $u_{g} r=\alpha_{g}\left(r 1_{g^{-1}}\right) u_{g}$, for all $r \in R, g \in G$.

- $B y\left[17\right.$, Lemma 6.7] the map $J_{g} \otimes D_{h} \stackrel{\kappa_{g, h}}{\rightarrow} D_{g h} \otimes J_{g}$ such that

$$
a_{g} \otimes b_{h} \mapsto \alpha_{g}\left(b_{h} 1_{g^{-1}}\right) \otimes a_{g},
$$

for any $g, h \in G$, is a $R$-R-bimodule isomorphism.

The construction of $\varphi_{6}$. Take $f \in Z^{1}\left(G, \alpha^{*}, \mathbf{P i c S}(R)\right)$. By the second item of Proposition 6.2 there exists a family of $R$ - $R$-bimodule isomorphisms $\left\{f_{g, h}: J_{g} \otimes J_{h} \rightarrow\right.$ $\left.D_{g} \otimes J_{g h}\right\}_{g, h \in G}$. Consider the following diagram

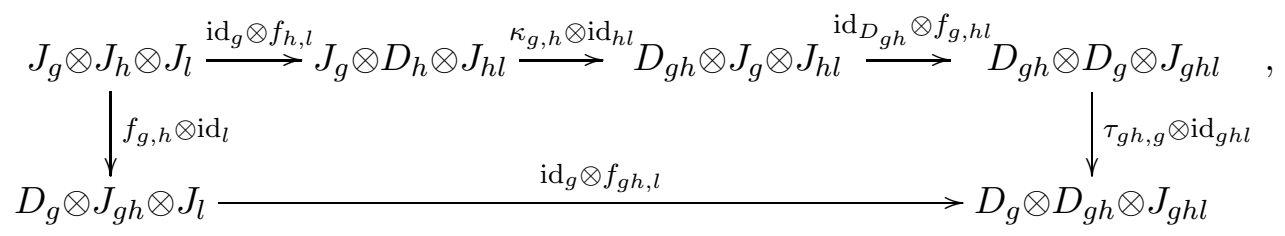

for any $g, h, l \in G$, where $\kappa_{g, h}$ is given by the second item of Remark 6.3. Set $\tilde{\omega}(g, h, l)=$ $\left(\mathrm{id}_{g} \otimes f_{g h, l}\right) \circ\left(f_{g, h} \otimes \mathrm{id}_{l}\right) \circ\left(\mathrm{id}_{g} \otimes f_{h, l}\right)^{-1} \circ\left(\kappa_{g, h} \otimes \mathrm{id}_{h l}\right)^{-1} \circ\left(\mathrm{id}_{D_{g h}} \otimes f_{g, h l}\right)^{-1} \circ\left(\tau_{g h, g} \otimes \mathrm{id}_{g h l}\right)^{-1}$.

Then,

$$
\tilde{\omega}(g, h, l) \in \mathcal{U}\left(\operatorname{End}_{D_{g} \otimes D_{g h} \otimes D_{g h l}}\left(D_{g} \otimes D_{g h} \otimes J_{g h l}\right)\right),
$$

and there is a unique $\omega_{1}(g, h, l) \in \mathcal{U}\left(D_{g} \otimes D_{g h} \otimes D_{g h l}\right)$ such that $\tilde{\omega}(g, h, l)(z)=\omega_{1}(g, h, l) z$ for all $z \in D_{g} \otimes D_{g h} \otimes J_{g h l}$. This implies that there is a unique element $\omega_{f}(g, h, l) \in$ $\mathcal{U}\left(D_{g} D_{g h} D_{g h l}\right)$ with

$$
\tilde{\omega}(g, h, l) z=\omega_{f}(g, h, l) z, g, h, l \in G, z \in D_{g} \otimes D_{g h} \otimes J_{g h l} .
$$

Proposition 6.4. $\left[17\right.$, Theorem 6.9] The map $H^{1}\left(G, \alpha^{*}, \mathbf{P i c S}(R)\right) \ni \operatorname{cls}(f) \stackrel{\varphi_{6}}{\rightarrow} \operatorname{cls}\left(\omega_{f}\right) \in$ $H^{3}(G, \alpha, R)$ is a homomorphism of groups. 


\section{Partial generalized Crossed products and exactnesss at $H^{1}\left(G, \alpha^{*}, \operatorname{PicS}(R)\right)$}

Let $f \in Z^{1}\left(G, \alpha^{*}, \operatorname{PicS}(R)\right)$ and $\Phi_{f}=f \Phi_{0}$. Put $\Phi_{f}(g)=\left[J_{g}\right], g \in G$, and suppose that the diagram (26) given by the family of $R$ - $R$-bimodule isomorphisms $\mathfrak{F}=$ $\left\{f_{g, h}: J_{g} \otimes J_{h} \rightarrow D_{g} \otimes J_{g h}\right\}_{g, h \in G}$ commutes. Following [23] we call $\mathfrak{F}$ a factor set of $G$ related to $\Phi=\Phi_{f}$. Consider the $R$ - $R$-bimodule

$$
\Delta=\Delta(\mathfrak{F}, \alpha, R, \Phi, G)=\bigoplus_{g \in G} J_{g} .
$$

Let $m_{g, h}: D_{g} \otimes J_{g h} \rightarrow D_{g} J_{g h} \subseteq J_{g h}, g, h \in G$, be the multiplication map ${ }^{2}$. The product of elements in $\Delta$ is defined by the formula $x y=m_{g, h} \circ f_{g, h}(x \otimes y)$, for any $x \in J_{g}, y \in J_{h}$.

We call $\Delta(\mathfrak{F}, \alpha, R, \Phi, G)$ a partial generalized crossed product. Notice that for any $x \in J_{g}, y \in J_{h}$, and $f_{g, h}(x \otimes y)=\sum d_{g} \otimes u_{g h}$ we have $x y=\sum d_{g} u_{g h}$. From this we obtain

$$
f_{g, h}(x \otimes y)=1_{g} \otimes \sum d_{g} u_{g h}=1_{g} \otimes x y .
$$

In particular, for any $r \in R, r(x y)=\sum r d_{g} u_{g h}=m_{g, h} \circ f_{g, h}(r x \otimes y)=(r x) y$, and $(x y) r=m_{g, h} \circ f_{g, h}(x \otimes y r)=x(y r)$. We conclude that

$$
r(x y)=(r x) y, \text { and }(x y) r=x(y r) \text { for any } g, h \in G, r \in R, x \in J_{g} \text { and } y \in J_{h} .
$$

Proposition 7.1. The partial generalized crossed product $\Delta=\Delta(\mathfrak{F}, \alpha, R, \Phi, G)$ is an associative $R^{\alpha}$-algebra with identity element and $J_{1} \cong R$ as $R$-algebras.

Proof. Let $x \in J_{g}, y \in J_{h}, z \in J_{l}$. Then

$$
\begin{aligned}
1_{g} \otimes 1_{g h} \otimes x(y z) & =\left(\tau_{g h, g} \otimes \iota_{g h l}\right)\left(1_{g h} \otimes 1_{g} \otimes x(y z)\right) \\
& \stackrel{(27)}{=}\left(\tau_{g h, g} \otimes \iota_{g h l}\right)\left(1_{g h} \otimes f_{g, h l}(x \otimes y z)\right) \\
& =\left(\tau_{g h, g} \otimes \iota_{g h l}\right)\left(\mathrm{id}_{D_{g h}} \otimes f_{g, h l}\right)\left(1_{g h} \otimes x \otimes y z\right) \\
& \stackrel{x \in J_{g}}{=}\left(\tau_{g h, g} \otimes \iota_{g h l}\right)\left(\mathrm{id}_{D_{g h}} \otimes f_{g, h l}\right)\left(1_{g} 1_{g h} \otimes x \otimes y z\right) \\
& =\left(\tau_{g h, g} \otimes \iota_{g h l}\right)\left(\mathrm{id}_{D_{g h}} \otimes f_{g, h l}\right)\left(\kappa_{g, h} \otimes \iota_{h l}\right)\left(x \otimes 1_{h} \otimes y z\right) \\
& \stackrel{(27)}{=}\left(\tau_{g h, g} \otimes \iota_{g h l}\right)\left(\mathrm{id}_{D_{g h}} \otimes f_{g, h l}\right)\left(\kappa_{g, h} \otimes \iota_{h l}\right)\left(\iota_{g} \otimes f_{h, l}\right)(x \otimes y \otimes z) \\
& \stackrel{(26)}{=}\left(\mathrm{id}_{D_{g}} \otimes f_{g h, l}\right)\left(f_{g, h} \otimes \iota_{l}\right)(x \otimes y \otimes z) \\
& =\left(\mathrm{id}_{D_{g}} \otimes f_{g h, l}\right)\left(f_{g, h}(x \otimes y) \otimes z\right) \\
& \stackrel{(27)}{=}\left(\mathrm{id}_{D_{g}} \otimes f_{g h, l}\right)\left(1_{g} \otimes x y \otimes z\right) \\
& =1_{g} \otimes f_{g h, l}(x y \otimes z) \\
& \stackrel{(27)}{=} 1_{g} \otimes 1_{g h} \otimes(x y) z,
\end{aligned}
$$

for any $g, h \in G$, which implies $\left(1_{g} 1_{g h}\right)[x(y z)]=\left(1_{g} 1_{g h}\right)[(x y) z]$. Furthermore, since $x \in J_{g}$ and $y \in J_{h}$, using (28) we have

\footnotetext{
${ }^{2}$ Notice that if $g=1$ or $h=1$ then $m_{g, h}$ is an $R$ - $R$-bimodule isomorphism.
} 


$$
\begin{aligned}
\left(1_{g} 1_{g h}\right)[x(y z)] & =\left(1_{g h} x\right)(y z) \\
& \stackrel{(25)}{=}\left(x 1_{h}\right)(y z) \\
& =\left(m_{g, h l} \circ f_{g, h l}\right)\left(x 1_{h} \otimes y z\right) \\
& =m_{g, h l} \circ f_{g, h l}(x \otimes y z) \\
& =x(y z) .
\end{aligned}
$$

Moreover, since $x y \in J_{g h}$, we get

$$
\begin{aligned}
\left(1_{g} 1_{g h}\right)[(x y) z] & \stackrel{(28)}{=}\left[\left(1_{g} 1_{g h}\right)(x y)\right] z \\
& =\left[\left(1_{g}(x y)\right] z\right. \\
& \stackrel{(28)}{=}\left[\left(1_{g} x\right) y\right] z \\
& =(x y) z
\end{aligned}
$$

and $\Delta$ is associative.

Now we will check that $J_{1} \cong R$ as $R$-algebras. For $x, y \in J_{1}, x y=m_{1,1} f_{1,1}(x \otimes y) \in J_{1}$, and $J_{1}$ is closed by products. Moreover, $\left[J_{1}\right]=\Phi(1)=[R]$, and $J_{1} \cong R$ as $R$-Rbimodules. Let $\phi: R \rightarrow J_{1}$ be an $R$ - $R$-bimodule isomorphism. Denoting $u=\phi(1)$ we have $J_{1}=\phi(R)=R u=u R$, and it follows from (25) that $u r=r u$, for any $r \in R$.

Since $m_{1,1} \circ f_{1,1}$ is a $R$ - $R$-bimodule isomorphism, $u \otimes u$ is a basis of $J_{1} \otimes J_{1}$ and then there exists $c \in \mathcal{U}(R)$ such that $m_{1,1} \circ f_{1,1}(u \otimes u)=c u$. If we set $e=c^{-1} u \in J_{1}$, then $J_{1}=e R, e e=m_{1,1} \circ f_{1,1}\left(c^{-1} u \otimes c^{-1} u\right)=e$ and the map $R \ni r \mapsto r e \in J_{1}$ is a $R$-module isomorphism.

The element $e$ is the identity of $\Delta$. Indeed, let $x \in J_{l}, l \in G$. Since $m_{1, l} \circ f_{1, l}: J_{1} \otimes J_{l} \cong J_{l}$ is a $R$ - $R$-bimodule isomorphism, there exists $\sum_{i} r_{i} e \otimes y_{i} \in J_{1} \otimes J_{l}$, such that $x=m_{1, l} \circ$ $f_{1, l}\left(\sum_{i} r_{i} e \otimes y_{i}\right)$. Writing $y=\sum_{i} r_{i} y_{i} \in J_{l}$ we have that $\sum_{i} r_{i} e \otimes y_{i}=e \otimes y$ and $x=m_{1, l} \circ$ $f_{1, l}(e \otimes y)=e y$. Analogously using the $R$ - $R$-bimodule isomorphism $m_{l, 1} \circ f_{l, 1}: J_{l} \otimes J_{1} \cong J_{l}$ we see that there exists $y^{\prime} \in J_{l}$ such that $x=y^{\prime} e$. Then $e x=e(e y)=(e e) y=e y=x$ and $x e=\left(y^{\prime} e\right) e=y^{\prime} e=x$.

Therefore, $\Delta$ is an $R^{\alpha}$-algebra with $1_{\Delta}=e$, and the map $R \ni r \mapsto r e \in J_{1}$ is multiplicative, which yields that $J_{1} \cong R$ as $R$-algebras.

Example 7.2. Suppose that $f(g)=\left[D_{g}\right]$ for all $g \in G$. Then $\Delta(\mathfrak{F}, \alpha, R, \Phi, G)$ is a partial crossed product with respect to a twisting of $\alpha$. Indeed, as in Remark 6.3 we have $J_{g}=D_{g} u_{g}$, where $u_{g} r=\alpha_{g}\left(r 1_{g^{-1}}\right) u_{g}$, and $u_{g}$ is a free $D_{g^{-b a s i s}}$ of $J_{g}$ for all $g \in G$. Write $f_{g, h}\left(u_{g} \otimes u_{h}\right)=\omega_{g, h} \otimes u_{g h} \in D_{g} D_{g h} \otimes u_{g h}=D_{g} \otimes D_{g h} u_{g h}$. Then, $\omega_{g, h} \in \mathcal{U}\left(D_{g} D_{g h}\right)$. In fact, since $f_{g, h}$ is an isomorphism of $R$-R-bimodules, there exists $x=\sum_{i} a_{g}^{i} u_{g} \otimes b_{h}^{i} u_{h} \in$ $D_{g} u_{g} \otimes D_{h} u_{h}$ with $f_{g, h}(x)=1_{g} 1_{g h} \otimes u_{g h}$. Then,

$$
x=\sum_{i} a_{g}^{i} u_{g} b_{h} \otimes u_{h} \stackrel{(25)}{=} \sum_{i} a_{g}^{i} \alpha_{g}\left(b_{h}^{i} 1_{g^{-1}}\right) u_{g} \otimes u_{h}=x_{g, h} u_{g} \otimes u_{h},
$$


where $x_{g, h}=\sum_{i} a_{g}^{i} \alpha_{g}\left(b_{h}^{i} 1_{g^{-1}}\right) \in D_{g} D_{g h}$. Consequently,

$$
1_{g} 1_{g h} \otimes u_{g h}=f_{g, h}(x)=x_{g, h} f_{g, h}\left(u_{g} \otimes u_{h}\right)=x_{g, h} \omega_{g, h} \otimes u_{g h},
$$

which gives $x_{g, h} \omega_{g, h} u_{g h}=1_{g} 1_{g h} u_{g h}$. Since $u_{g h}$ is a free generator of $J_{g h}$ over $D_{g h}$, we conclude that $x_{g, h} \omega_{g, h}=1_{g} 1_{g h}$, which shows that $\omega_{g, h} \in \mathcal{U}\left(D_{g} D_{g h}\right)$.

Notice that

$$
u_{g} u_{h}=m_{g, h} \circ f_{g, h}\left(u_{g} \otimes u_{h}\right)=\omega_{g, h} u_{g h} .
$$

Since $\left(u_{g} u_{h}\right) u_{l}=u_{g}\left(u_{h} u_{l}\right)$, for any $g, h, l \in G$, it follows that the map $\omega: G \times G \ni$ $(g, h) \rightarrow \omega_{g, h} \in R$ belongs to $Z^{2}(G, \alpha, R)$. We conclude by (25) that $\Delta \cong \bigoplus_{g \in G} D_{g} u_{g}=$ $R \star_{\alpha, \omega} G$ as $R$-R-bimodules and $R^{\alpha}$-algebras.

By Proposition 7.1 one may identify $R \cong J_{1} \subseteq \Delta$. In particular, $1_{R}=1_{\Delta}$. This will be assumed in all what follows.

Our next result states that every partial generalized crossed product is an Azumaya $R^{\alpha}$-algebra which is split by $R$.

Proposition 7.3. Let $f \in Z^{1}\left(G, \alpha^{*}, \operatorname{PicS}(R)\right), \Phi=f \Phi_{0}$ and $\mathfrak{F}$ a factor set of $G$ related to $\Phi$. Then the partial generalized crossed product $\Delta=\Delta(\mathfrak{F}, \alpha, R, \Phi, G)$ is an Azumaya $R^{\alpha}$-algebra containing $R$ as a maximal commutative subalgebra.

Proof. For $h \in G$, we see that

$$
J_{h}=\left\{z \in \Delta \mid z r=\alpha_{h}\left(r 1_{h^{-1}}\right) z, \text { for all } r \in R\right\} .
$$

Indeed, fix $h \in G$. Then, for any $z=\sum_{g \in G} z_{g} \in \Delta$, satisfying $z r=\alpha_{h}\left(r 1_{h^{-1}}\right) z$ for all $r \in R$, we have

$$
\sum_{g \in G} \alpha_{h}\left(r 1_{h^{-1}}\right) z_{g}=\alpha_{h}\left(r 1_{h^{-1}}\right) z=z r=\sum_{g \in G} z_{g} r=\sum_{g \in G} \alpha_{g}\left(r 1_{g^{-1}}\right) z_{g},
$$

and

$$
\alpha_{h}\left(r 1_{h^{-1}}\right) z_{g}=\alpha_{g}\left(r 1_{g^{-1}}\right) z_{g},
$$

for all $r \in R, g \in G$. In particular, for $r_{h}=\alpha_{h^{-1}}\left(r 1_{h}\right)$ we obtain

$$
\left(r 1_{h}\right) z_{g}=\alpha_{h}\left(r_{h}\right) z_{g}=\alpha_{g}\left(r_{h} 1_{g^{-1}}\right) z_{g} \stackrel{(4)}{=} \alpha_{g h^{-1}}\left(r 1_{h g^{-1}}\right) 1_{g} z_{g}=\alpha_{g h^{-1}}\left(r 1_{h g^{-1}}\right) z_{g},
$$

for any $g \in G$ and any $r \in R$. Since $R / R^{\alpha}$ is a partial Galois extension, there exists a family $\left\{x_{i}, y_{i} \mid 1 \leq i \leq m\right\} \subseteq R$, such that $\sum_{i=1}^{m} x_{i} \alpha_{g h^{-1}}\left(y_{i} 1_{h g^{-1}}\right)=\delta_{1, g h^{-1}}$, for any $g \in G$. Thus for $g \neq h$,

$$
1_{h} z_{g}=\sum_{i=1}^{m} x_{i} y_{i}\left(1_{h} z_{g}\right)=\sum_{i=1}^{m} x_{i}\left[\left(y_{i} 1_{h}\right) z_{g}\right]=\sum_{i=1}^{m} x_{i} \alpha_{g h^{-1}}\left(y_{i} 1_{h g^{-1}}\right) z_{g}=0,
$$

and $1_{h} z_{g}=0$. From this we get

$$
z_{g}=1_{g} z_{g}=\alpha_{g}\left(1_{R} 1_{g^{-1}}\right) z_{g} \stackrel{(30)}{=} \alpha_{h}\left(1_{R} 1_{h^{-1}}\right) z_{g}=1_{h} z_{g}=0 .
$$


Therefore $z=z_{h} \in J_{h}$ and using (25) we obtain (29). In particular, if $z \in C_{\Delta}(R)$ we have $z r=r z$ for any $r \in R$, then $z \in J_{1}=R$. Thus $C_{\Delta}(R)=R$.

Now we prove that $R^{\alpha}=C(\Delta)$. We have $R^{\alpha} \subseteq C(\Delta)=C_{\Delta}(\Delta) \subseteq C_{\Delta}(R)=R$. Let $r \in C(\Delta)$. Then for $x \in J_{g}$ we have $r x=x r=\alpha_{g}\left(r 1_{g^{-1}}\right) x$, which implies $r 1_{g} x=$ $1_{g}(r x)=\alpha_{g}\left(r 1_{g^{-1}}\right) x$. Notice that $D_{g} \cong \operatorname{End}_{D_{g}}\left(J_{g}\right)$, implies that $J_{g}$ is a faithful $D_{g^{-}}$ module. Then $r 1_{g}=\alpha_{g}\left(r 1_{g^{-1}}\right)$, for all $g \in G$, and therefore $r \in R^{\alpha}$.

Finally we show that $\Delta / R^{\alpha}$ is separable. By [7, Theorem 2.7.1] it is enough to prove that $\Delta_{\mathfrak{m}} /\left(R^{\alpha}\right)_{\mathfrak{m}}$ is separable, for every maximal ideal $\mathfrak{m}$ of $R^{\alpha}$. But if $R^{\alpha}$ is local, then $R$ is semi-local being a f.g.p. over $R^{\alpha}$. It follows that $\operatorname{Pic}(R)=0$, and hence $\operatorname{Pic}\left(D_{g}\right)=0$, for all $g \in G$ as $D_{g}$ is a direct factor of $R$. Thanks to the third item of Lemma $3.8 f$ is locally trivial, i.e. $f(g)_{\mathfrak{p}} \cong\left(D_{g}\right)_{\mathfrak{p}}$, for any $\mathfrak{p} \in \operatorname{Spec}(R)$, and it follows from Lemma 3.7 that $f(g) \in \operatorname{Pic}\left(D_{g}\right)=0$, so that $f(g) \cong D_{g}$ as $D_{g}$-modules as well as $R$-modules. Then, Example 7.2 implies that $\Delta_{\mathfrak{m}}$ is a crossed product by a twisted partial action, and consequently, $\Delta_{\mathfrak{m}}$ is separable over $R^{\alpha}$ thanks to [27, Proposition 3.2].

Remark 7.4. It is readily seen that the ring isomorphism $\alpha_{g}: D_{g^{-1}} \rightarrow D_{g}$ gives an $R$-R-bimodule isomorophism

$$
{ }_{g}\left(D_{g^{-1}}\right)_{I} \cong_{I}\left(D_{g}\right)_{g^{-1}},
$$

where ${ }_{I}\left(D_{g}\right)_{g^{-1}}=D_{g}$, as sets, the left $R$-action on ${ }_{I}\left(D_{g}\right)_{g^{-1}}$ is $(r, d) \mapsto r d$ and the right $R$-action is defined by $(d, r) \mapsto d \alpha_{g}(r), a \in D_{g}, r \in R$. Then obviously $\Phi_{0}(g)=$ $\left[{ }_{g}\left(D_{g^{-1}}\right)_{I}\right]=\left[{ }_{I}\left(D_{g}\right)_{g^{-1}}\right] \in \mathbf{P i c S}_{R^{\alpha}}(R)$.

Proposition 7.5. Let $A$ be an Azumaya $R^{\alpha}$-algebra containing $R$ as a maximal commutative subalgebra. Take $f \in Z^{1}\left(G, \alpha^{*}, \mathbf{P i c S}(R)\right)$ such that $\varphi_{5}([A])=\operatorname{cls}(f)$. Write $\Phi_{f}(g)=\left[J_{g}\right]$, then there is a factor set $\mathcal{F}=\left\{f_{g, h}: J_{g} \otimes J_{h} \rightarrow D_{g} \otimes J_{g h}\right\}$ for $\Phi_{f}$ and $\Delta=\Delta\left(\mathfrak{F}, \alpha, R, \Phi_{f}, G\right)=\bigoplus_{g \in G} J_{g}$ is a partial generalized crossed product.

Proof. Write $f(g)=\left[C_{g}\right]$ for all $g \in G$, then $J_{g} \simeq C_{g} \otimes_{I}\left(D_{g}\right)_{g^{-1}}$. By Remark 5.4 one may assume that

$$
C_{g}=\left\{a \in 1_{g^{-1}} A \mid \alpha_{g^{-1}}\left(r 1_{g}\right) a=a r, \text { for any } r \in R\right\},
$$

with left and right $R$-module actions given by $r \bullet a=\alpha_{g^{-1}}\left(r 1_{g}\right) a=a r=a \bullet r$, for $r \in R$ and $a \in C_{g}$. Let $J_{g}^{\prime}=C_{g}$ as sets and suppose that the left and right $R$-module actions on $J_{g}^{\prime}$ are given by

$$
r \star a=\alpha_{g^{-1}}\left(r 1_{g}\right) a \text { and } a \star r=a \alpha_{g}\left(r 1_{g^{-1}}\right),
$$

for $r \in R$ and $a \in J_{g}^{\prime}$.

Consider the $R$ - $R$-bimodule map

$$
\begin{array}{rlr}
\lambda: J_{g}^{\prime} & \longrightarrow & C_{g} \otimes_{I}\left(D_{g}\right)_{g^{-1}} \\
u_{g} & \longmapsto & u_{g} \otimes 1_{g}
\end{array},
$$

and let

$$
\begin{aligned}
\zeta: C_{g} \otimes_{I}\left(D_{g}\right)_{g^{-1}} & \longrightarrow J_{g}^{\prime}, \\
c_{g} \otimes d_{g} & \longmapsto c_{g} d_{g} .
\end{aligned}
$$


Then it is not difficult to check that $\zeta=\lambda^{-1}$, and $\lambda$ is an isomorphism, so $J_{g}^{\prime} \simeq J_{g}$ when considered with the left and right $R$-module actions given by (31).

On the other hand, by Proposition 6.2 the map $\Phi_{f}$ is a partial representation, and we shall construct a factor set for it.

Let $u_{g} \in J_{g}, u_{h} \in J_{h}$ and $r \in R$, then

$$
\begin{aligned}
\alpha_{(g h)^{-1}}\left(r 1_{g h}\right) u_{h} h_{g} & =\alpha_{(g h)^{-1}}\left(r 1_{g h}\right) 1_{h^{-1}} u_{h} h_{g} \\
& =\alpha_{h^{-1}}\left(\alpha_{g^{-1}}\left(r 1_{g}\right) 1_{h}\right) u_{h} u_{g} \\
& =u_{h} \alpha_{g^{-1}}\left(r 1_{g}\right) u_{g} \\
& =u_{h} u_{g} r .
\end{aligned}
$$

In particular,

$$
1_{(g h)^{-1}} u_{h} u_{g}=\alpha_{(g h)^{-1}}\left(1_{g} 1_{g h}\right) u_{h} u_{g}=u_{h} u_{g} 1_{g}=u_{h} u_{g} .
$$

Thus $u_{h} u_{g} \in J_{g h}$ and the map

$$
\begin{aligned}
f_{g, h}: J_{g} \otimes J_{h} & \longrightarrow D_{g} \otimes J_{g h} \\
u_{g} \otimes u_{h} & \longmapsto 1_{g} \otimes u_{h} u_{g}
\end{aligned},
$$

is well defined, and it is not difficult to show that $f_{g, h}$ is $R$-bilinear. Now we check that $f_{g, h}$ is an isomorphism. Localizing by an ideal in $\operatorname{Spec}\left(R^{\alpha}\right)$ we may assume that $R^{\alpha}$ is a local ring. This implies that $R$ is semilocal and $\operatorname{Pic}(R)=\{1\}$. Since $D_{g}$ is a direct summand of $R$, we obtain $\operatorname{Pic}\left(D_{g}\right)=\{1\}$. But $\left[C_{g}\right] \in \operatorname{Pic}\left(D_{g}\right)$, and there is a $R$ - $R$-bimodule isomorphism $\gamma_{g}: D_{g} \longrightarrow C_{g}$. Set $\omega_{g}=\gamma_{g}\left(1_{g}\right) \in C_{g}$, then given $c_{g} \in C_{g}$, there exists $d \in D_{g}$ such that

$$
c_{g}=\gamma_{g}(d)=d \cdot \gamma_{d}\left(1_{g}\right)=\alpha_{g^{-1}}(d) \omega_{g}=\omega_{g} d .
$$

This implies that $C_{g}=D_{g} \cdot \omega_{g}=\omega_{g} D_{g}$ with $\alpha_{g^{-1}}\left(r 1_{g^{-1}}\right) \omega_{g}=\omega_{g} r$, for each $r \in R$.

Consider the isomorphism $\gamma_{g}^{\prime}:{ }_{I}\left(D_{g}\right)_{g^{-1}} \rightarrow J_{g}$ given by the composition

$$
{ }_{I}\left(D_{g}\right)_{g^{-1}} \longrightarrow D_{g} \otimes_{I}\left(D_{g}\right)_{g^{-1}} \longrightarrow C_{g} \otimes_{I}\left(D_{g}\right)_{g^{-1}} \longrightarrow J_{g}
$$

Then $\gamma_{g}^{\prime}\left(1_{g}\right)=\omega_{g}$, and $J_{g}=D_{g^{-1}} \omega_{g}=\omega_{g} D_{g}$. From this we get that

$$
\begin{aligned}
f_{g, h}: \omega_{g} D_{g} \otimes \omega_{h} D_{h} & \longrightarrow D_{g} \otimes \omega_{g h} D_{g h} \\
\omega_{g} d_{g} \otimes \omega_{h} d_{h} & \longmapsto 1_{g} \otimes \omega_{h} d_{h} \omega_{g} d_{g},
\end{aligned}
$$

for all $g, h \in G$. Now we construct the inverse of $f_{g, h}$. By Lemma 5.3, there is an isomorphism $_{g}\left(1_{g^{-1}} A\right) \simeq C_{g} \otimes A$. Localizing by an ideal in $\operatorname{Spec}\left(R^{\alpha}\right)$, we have $g_{g}\left(1_{g^{-1}} A\right) \simeq$ $\omega_{g} D_{g} \otimes A \simeq \omega_{g} A$. Since $1_{g^{-1}} \in 1_{g^{-1}} A$, there is $a_{g} \in A$ such that

$$
\omega_{g} a_{g}=1_{g^{-1}}
$$

But

$$
\omega_{g}\left(a_{g} \omega_{g}\right)=\left(\omega_{g} a_{g}\right) \omega_{g}=1_{g^{-1}} \omega_{g}=\omega_{g} 1_{g}
$$

Since $\omega_{g}$ is a free basis of $g_{g}\left(1_{g^{-1}} A\right)$, we obtain

$$
a_{g} \omega_{g}=1_{g}
$$


For $r \in R$, one has that

$$
\begin{aligned}
\omega_{g} \alpha_{g}\left(r 1_{g^{-1}}\right) a_{g} & =r \omega_{g} a_{g}=r 1_{g^{-1}} \\
& =1_{g^{-1}} r=\omega_{g} a_{g} r .
\end{aligned}
$$

Then,

$$
\alpha_{g}\left(r 1_{g^{-1}}\right) a_{g}=a_{g} r, \text { for all } r \in R
$$

But

$$
\begin{aligned}
1_{g} a_{g} a_{h} 1_{h^{-1}} \omega_{g h} r & =1_{g} a_{g} a_{h} 1_{h^{-1}} \alpha_{h^{-1} g^{-1}}\left(r 1_{g h}\right) \omega_{g h} \\
& =1_{g} a_{g} a_{h} 1_{h^{-1}} \alpha_{h^{-1}}\left(\alpha_{g^{-1}}\left(r 1_{g}\right) 1_{h^{-1}}\right) \omega_{g h} \\
& \stackrel{(34)}{=} 1_{g} a_{g} \alpha_{g^{-1}}\left(r 1_{g}\right) a_{h} 1_{h^{-1}} \omega_{g h} \\
& \stackrel{(34)}{=} r 1_{g} a_{g} a_{h} 1_{h^{-1}} \omega_{g h} .
\end{aligned}
$$

Then, $1_{g} a_{g} a_{h} 1_{h^{-1}} \omega_{g h} \in C_{A}(R)=R$. Hence, $1_{g} a_{g} a_{h} 1_{h^{-1}} \omega_{g h}=1_{g} 1_{g h} a_{g} a_{h} \omega_{g h} \in D_{g} D_{g h}$. Finally, we see that

$$
\begin{aligned}
\omega_{h} \omega_{g}\left(1_{g} a_{g} a_{h} 1_{h^{-1}} \omega_{g h}\right) & =\omega_{g} a_{g} a_{h} 1_{h^{-1}} \omega_{g h} \\
& \stackrel{(32)}{=} \omega_{h} 1_{g^{-1}} a_{h} 1_{h^{-1}} \omega_{g h} \\
& =\alpha_{h^{-1}}\left(1_{g^{-1}} 1_{h}\right) \omega_{h} a_{h} 1_{h^{-1}} \omega_{g h} \\
& \stackrel{(32)}{=} 1_{h^{-1} g^{-1}} 1_{h^{-1}} \omega_{g h} \\
= & 1_{h^{-1}} \omega_{g h} .
\end{aligned}
$$

We consider the well defined map

$$
\begin{aligned}
l_{g, h}: D_{g} \otimes \omega_{g h} D_{g h} & \longrightarrow \omega_{g} D_{g} \otimes \omega_{h} D_{h} \\
d_{g} \otimes \omega_{g h} d_{g h} & \longmapsto \omega_{g}\left(d_{g} a_{g} a_{h} 1_{h^{-1}} \omega_{g h} d_{g h}\right) \otimes \omega_{h} .
\end{aligned}
$$


Moreover,

$$
\begin{aligned}
& l_{g, h} \circ f_{g, h}\left(\omega_{g} d_{g} \otimes \omega_{h} d_{h}\right)=\quad=\quad l_{g, h}\left(1_{g} \otimes \omega_{h} d_{h} \omega_{g} d_{g}\right) \\
& =l_{g, h}\left(1_{g} \otimes \omega_{h} \omega_{g} \alpha_{g}\left(d_{h} 1_{g^{-1}}\right) d_{g}\right) \\
& =\omega_{g}\left(1_{g} a_{g} a_{h} 1_{h^{-1}} \omega_{h} \omega_{g} \alpha_{g}\left(d_{h} 1_{g^{-1}}\right) d_{g}\right) \otimes \omega_{h} \\
& =\omega_{g} a_{g} a_{h} \omega_{h} \omega_{g} d_{g} \alpha_{g}\left(d_{h} 1_{g^{-1}}\right) \otimes \omega_{h} \\
& \stackrel{(33),(32)}{=} 1_{g^{-1}} 1_{h} \omega_{g} d_{g} \alpha_{g}\left(d_{h} 1_{g^{-1}}\right) \otimes \omega_{h} \\
& =\quad \alpha_{g^{-1}}\left(1_{g h} 1_{g}\right) \omega_{g} d_{g} \alpha_{g}\left(d_{h} 1_{g^{-1}}\right) \otimes \omega_{h} \\
& =\omega_{g} 1_{g h} d_{g} \alpha_{g}\left(d_{h} 1_{g^{-1}}\right) \otimes \omega_{h} \\
& =\omega_{g} d_{g} \alpha_{g}\left(1_{h} 1_{g^{-1}}\right) \alpha_{g}\left(d_{h} 1_{g^{-1}}\right) \otimes \omega_{h} \\
& =\omega_{g} d_{g} \alpha_{g}\left(d_{h} 1_{g^{-1}}\right) \otimes \omega_{h} \\
& =\omega_{g} d_{g} \cdot d_{h} \otimes \omega_{h} \\
& =\omega_{g} d_{g} \otimes d_{h} \cdot \omega_{h} \\
& =\omega_{g} d_{g} \otimes \alpha_{h^{-1}}\left(d_{h}\right) \omega_{h} \\
& =\omega_{g} d_{g} \otimes \omega_{h} d_{h},
\end{aligned}
$$

and

$$
\begin{aligned}
f_{g, h} \circ l_{g, h}\left(d_{g} \otimes \omega_{g h} d_{g h}\right) & =f_{g, h}\left(\omega_{g}\left(d_{g} a_{g} a_{h} 1_{h^{-1}} \omega_{g h} d_{g h}\right) \otimes \omega_{h}\right) \\
& =1_{g} \otimes \omega_{h} \omega_{g} d_{g} a_{g} a_{h} 1_{h^{-1}} \omega_{g h} d_{g h} \\
& =1_{g} \otimes \omega_{h} \alpha_{g^{-1}}\left(d_{g}\right) \omega_{g} a_{g} a_{h} 1_{h^{-1}} \omega_{g h} d_{g h} \\
& \stackrel{(32)}{=} 1_{g} \otimes \omega_{h} \alpha_{g^{-1}}\left(d_{g}\right) 1_{g^{-1}} a_{h} 1_{h^{-1}} \omega_{g h} d_{g h} \\
& =1_{g} \otimes \alpha_{h^{-1}}\left(\alpha_{g^{-1}}\left(d_{g}\right)\right) \omega_{h} a_{h} 1_{h^{-1}} \omega_{g h} d_{g h} \\
& \stackrel{(32)}{=} 1_{g} \otimes \alpha_{h^{-1}}\left(\alpha_{g^{-1}}\left(d_{g}\right)\right) 1_{h^{-1}} 1_{h^{-1}} \omega_{g h} d_{g h} \\
& =1_{g} \otimes \alpha_{h^{-1} g^{-1}}\left(d_{g} 1_{g h}\right) \omega_{g h} d_{g h} \\
& =1_{g} \otimes d_{g} \cdot \omega_{g h} d_{g h} \\
& =d_{g} \otimes \omega_{g h} d_{g h} .
\end{aligned}
$$

and we conclude that $f_{g, h}$ is an isomorphism. Thus to show that $\mathcal{F}=\left\{f_{g, h}: J_{g} \otimes J_{g} \longrightarrow\right.$ $\left.D_{g} \otimes J_{g h}, g, h \in G\right\}$ is a factor set, it only remains to prove that the diagram

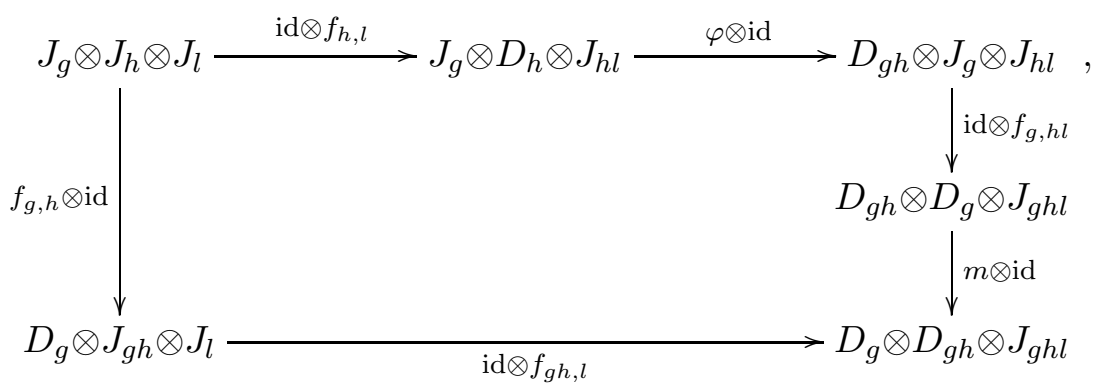


where

$$
\begin{array}{ccc}
\varphi: J_{g} \otimes D_{h} & \longrightarrow & D_{g h} \otimes J_{g} \\
u_{g} \otimes d_{h} & \longmapsto \alpha_{g}\left(d_{h} 1_{g^{-1}}\right) \otimes u_{g}
\end{array}
$$

and

$$
\begin{aligned}
m: D_{g h} \otimes D_{g} & \longrightarrow D_{g} \otimes D_{g h} \\
d_{g h} \otimes d_{g} & \longmapsto d_{g} \otimes d_{g h}
\end{aligned}
$$

is commutative. Indeed, for $u_{g} \in J_{g}, u_{h} \in J_{h}, u_{l} \in J_{l}$, we have

$$
\begin{aligned}
u_{g} \otimes u_{h} \otimes u_{l} \stackrel{f_{g, h} \otimes \mathrm{id}}{\longmapsto} & 1_{g} \otimes u_{h} u_{g} \otimes u_{l} \\
\stackrel{\mathrm{id} \otimes f_{g h, l}}{\longmapsto} & 1_{g} \otimes 1_{g h} \otimes u_{l} u_{h} u_{g} .
\end{aligned}
$$

and,

$$
\begin{array}{rll}
u_{g} \otimes u_{h} \otimes u_{l} & \stackrel{\mathrm{id} \otimes f_{h, l}}{\longmapsto} & u_{g} \otimes 1_{h} \otimes u_{l} u_{h} \\
& \stackrel{\varphi \otimes \mathrm{id}}{\longmapsto} & 1_{g h} \otimes u_{g} \otimes u_{l} u_{h} \\
\stackrel{D_{g h} \otimes f_{g}, h l}{\longmapsto} & 1_{g h} \otimes 1_{g} \otimes u_{l} u_{h} u_{g} \\
\stackrel{m \otimes \mathrm{id}}{\longmapsto} & 1_{g} \otimes 1_{g h} \otimes u_{l} y_{h} u_{g} .
\end{array}
$$

then the diagram is commutative and $\Delta=\bigoplus_{g \in G} J_{g}$ is a partial generalized crossed product.

Lemma 7.6. Let $f \in Z^{1}\left(G, \alpha^{*}, \operatorname{PicS}(R)\right)$ be such that $\Delta=\Delta\left(\mathcal{F}, \alpha, R, f \Phi_{0}, G\right)=$ $\bigoplus_{g \in G} J_{g}$ is a partial generalized crossed product. Then $\varphi_{5}([\Delta])=\operatorname{cls}\left(f^{-1}\right)$.

Proof. Write $f(g)=\left[M_{g}\right]$, then $J_{g} \simeq M_{g} \otimes_{I}\left(D_{g}\right)_{g^{-1}}$. Since $M_{g}$ is a $D_{g}$-module, we have that $J_{g} \otimes_{I}\left(D_{g^{-1}}\right)_{g} \simeq M_{g} \otimes_{I}\left(D_{g}\right)_{g^{-1}} \otimes_{I}\left(D_{g^{-1}}\right)_{g} \simeq M_{g} \otimes D_{g} \simeq M_{g}$. Hence,

$$
M_{g} \simeq J_{g} \otimes_{I}\left(D_{g^{-1}}\right)_{g}
$$

By Proposition 7.3 we have that $[\Delta] \in B\left(R / R^{\alpha}\right)$ and the equality

$$
J_{g}=\left\{a \in \Delta: \alpha_{g}\left(r 1_{g^{-1}}\right) a=a r, \text { for all } r \in R\right\},
$$

follows by $(29)$. Denote $\varphi_{5}(\Delta)=c l s\left(f^{\prime}\right)$, where $f^{\prime}(g)=\left[C_{g}\right]$. By Lemma 5.3 we obtain

$$
C_{g}=\left\{a \in 1_{g^{-1}} \Delta ; \alpha_{g^{-1}}\left(r 1_{g}\right) a=a r, \text { for all } r \in R\right\} .
$$

Consider the well defined $R$-bimodule map

$$
\begin{aligned}
\varphi: I_{I}\left(D_{g}\right)_{g^{-1}} \otimes J_{g^{-1}} & \longrightarrow C_{g} \\
d \otimes x_{g^{-1}} & \longmapsto \alpha_{g^{-1}}(d) x_{g^{-1}} .
\end{aligned}
$$

Then

$$
\begin{array}{ccc}
\xi: C_{g} & \longrightarrow & I_{(}\left(D_{g}\right)_{g^{-1}} \otimes J_{g^{-1}} \\
c_{g} & \longmapsto & 1_{g} \otimes c_{g}
\end{array}
$$

is the inverse of $\varphi$. Indeed, for $c_{g} \in C_{g}$ we have

$$
(\varphi \circ \xi)\left(c_{g}\right)=\varphi\left(1_{g} \otimes c_{g}\right)=\alpha_{g^{-1}}\left(1_{g}\right) c_{g}=1_{g^{-1}} c_{g}=c_{g}
$$


Moreover, for $d \in D_{g}$ and $x_{g^{-1}} \in J_{g^{-1}}$, we see that

$$
\begin{aligned}
(\xi \circ \varphi)\left(d \otimes x_{g^{-1}}\right) & =\xi\left(\alpha_{g^{-1}}(d) x_{g^{-1}}\right) \\
& =1_{g} \otimes x_{g^{-1}} \\
& =1_{g} \cdot \alpha_{g^{-1}}(d) \otimes x_{g^{-1}} \\
& =d \otimes x_{g^{-1}}
\end{aligned}
$$

and we get that $\varphi=\xi^{-1}$, as there is an isomorphism

$$
C_{g} \simeq_{I}\left(D_{g}\right)_{g^{-1}} \otimes J_{g^{-1}}
$$

Since $f \in Z^{1}\left(G, \alpha^{*}, \operatorname{PicS}(R)\right)$, then

$$
\alpha_{g}^{*}\left(f(h)\left[D_{g^{-1}}\right]\right) f(g h)^{-1} f(g)=\left[D_{g}\right]\left[D_{g h}\right], \quad \forall g, h \in G .
$$

In particular, taking $h=g^{-1}$ and using the fact $f(1)=[R]$, we obtain

$$
f(g)^{-1}=\alpha_{g}^{*}\left(f\left(g^{-1}\right)\left[D_{g^{-1}}\right]\right), \forall g \in G .
$$

Then

$$
\begin{aligned}
C_{g} & \simeq C_{g} \otimes D_{g} \stackrel{(36)}{\simeq}_{I}\left(D_{g}\right)_{g^{-1}} \otimes J_{g^{-1}} \otimes D_{g} \\
& \simeq I\left(D_{g}\right)_{g^{-1}} \otimes J_{g^{-1}} \otimes_{I}\left(D_{g}\right)_{g^{-1}} \otimes_{I}\left(D_{g^{-1}}\right)_{g} \\
& \stackrel{(35)}{\simeq} I_{g}\left(D_{g}\right)_{g^{-1}} \otimes M_{g^{-1}} \otimes_{I}\left(D_{g^{-1}}\right)_{g} \\
& \simeq \alpha_{g}^{*}\left(f\left(g^{-1}\right)\left[D_{g^{-1}}\right]\right) \\
& \stackrel{(37)}{\simeq} f(g)^{-1} .
\end{aligned}
$$

Since $\varphi_{5}([\Delta])=\operatorname{cls}\left(f^{\prime}\right)$, where $f^{\prime}(g)=\left[C_{g}\right]=\left[f(g)^{-1}\right]$, for all $g \in G$. Then $f^{\prime}=f^{-1}$ and $\varphi_{5}(\Delta)=\operatorname{cls}\left(f^{-1}\right)$.

Theorem 7.7. The sequence $B\left(R / R^{\alpha}\right) \stackrel{\varphi_{5}}{\rightarrow} H^{1}\left(G, \alpha^{*}, \operatorname{PicS}(R)\right) \stackrel{\varphi_{6}}{\rightarrow} H^{3}(G, \alpha, R)$ is exact.

Proof. Let $f \in Z^{1}\left(G, \alpha^{*}, \operatorname{PicS}(R)\right)$ be such that $\operatorname{cls}(f) \in \operatorname{Im}\left(\varphi_{5}\right)$, then there is $[A] \in B\left(R / R^{\alpha}\right)$ with $\varphi_{5}([A])=\operatorname{cls}(f)$. Write $f(g)=\left[C_{g}\right]$, it follows from Proposition 7.5 that $\Delta=\bigoplus_{g \in G} J_{g}$, where $J_{g}=C_{g} \otimes_{I}\left(D_{g}\right)_{g^{-1}}$ is a partial generalized crossed product. Then, $\varphi_{6}(\operatorname{cls}(f))$ is trivial in $H^{3}(G, \alpha, R)$ and consequently $\operatorname{cls}(f) \in \operatorname{ker}\left(\varphi_{6}\right)$, and we obtain that $\operatorname{Im}\left(\varphi_{5}\right) \subseteq \operatorname{ker}\left(\varphi_{6}\right)$.

On the other hand, if $f \in Z^{1}\left(G, \alpha^{*}, \mathbf{P i c S}(R)\right)$ satisfies $c l s(f) \in \operatorname{ker}\left(\varphi_{6}\right)$, then $\Delta=$ $\bigoplus_{g \in G} J_{g}$, where $J_{g}=f(g) \otimes_{I}\left(D_{g}\right)_{g^{-1}}$, is a partial generalized crossed product and $[\Delta] \in$ $B\left(R / R^{\alpha}\right)$. By Lema 7.6, we have that $\varphi_{5}(\Delta)=\operatorname{cls}\left(f^{-1}\right)$. Then $\operatorname{cls}\left(f^{-1}\right) \in \operatorname{Im}\left(\varphi_{5}\right)$. Since $\operatorname{Im}\left(\varphi_{5}\right)$ is a group we conclude that $c l s(f) \in \operatorname{Im}\left(\varphi_{5}\right)$, proving that the sequence is exact.

Summarizing, we have obtained the following seven-term exact sequence:

$$
\begin{aligned}
& 0 \rightarrow H^{1}(G, \alpha, R) \stackrel{\varphi_{1}}{\rightarrow} \mathbf{P i c}\left(R^{\alpha}\right) \stackrel{\varphi_{2}}{\rightarrow} \mathbf{P i c S}(R)^{\alpha^{*}} \cap \mathbf{P i c}(R) \stackrel{\varphi_{3}}{\rightarrow} H^{2}(G, \alpha, R) \stackrel{\varphi_{4}}{\rightarrow} B\left(R / R^{\alpha}\right) \stackrel{\varphi_{5}}{\rightarrow} \\
& H^{1}\left(G, \alpha^{*}, \operatorname{PicS}(R)\right) \stackrel{\rightarrow}{\rightarrow} H^{3}(G, \alpha, R) .
\end{aligned}
$$


Corollary 7.8. Hilbert's $90^{\text {th }}$ Theorem for partial actions. Let $R \supseteq R^{\alpha}$ be $a$ partial Galois extension. If $\mathbf{P i c}\left(R^{\alpha}\right)=0$, then $H^{1}(G, \alpha, R)=0$.

Corollary 7.9. Crossed Product Theorem for partial actions. Let $R$ be a commutative ring such that $\mathbf{P i c}(R)$ is trivial. If $\alpha$ is a unital partial action of a finite group $G$ on $R$ such that $R / R^{\alpha}$ is an $\alpha$-partial Galois extension, then there is a group isomorphism $H^{2}(G, \alpha, R) \cong B\left(R / R^{\alpha}\right)$ given by $\operatorname{cls}(\omega) \mapsto\left[R \star_{\alpha, \omega} G\right]$.

Proof. Since $\operatorname{Pic}(R)$ is trivial we conclude that $\operatorname{Pic}\left(D_{g}\right)$ is trivial for all $g \in G$. Then by the third item of Lemma 3.8 we obtain that $f(g)=\left[D_{g}\right]$ for all $f \in Z^{1}\left(G, \alpha^{*}, \mathbf{P i c S}(R)\right)$ which implies that $H^{1}\left(G, \alpha^{*}, \mathbf{P i c} \mathbf{S}(R)\right)$ is trivial and $\varphi_{4}$ is an isomorphism.

Corollary 7.10. [5] Let $G$ be a finite group acting globally on the commutative ring $R$ and suppose that $R / R^{G}$ is a Galois extension. Then there is a seven term exact sequence $0 \rightarrow H^{1}(G, \mathcal{U}(R)) \stackrel{\varphi_{1}}{\rightarrow} \mathbf{P i c}\left(R^{G}\right) \stackrel{\varphi_{2}}{\rightarrow} \mathbf{P i c}(R)^{G} \stackrel{\varphi_{3}}{\rightarrow} H^{2}(G, \mathcal{U}(R)) \stackrel{\varphi_{4}}{\rightarrow} B\left(R / R^{\alpha}\right) \stackrel{\varphi_{5}}{\longrightarrow}$ $H^{1}(G, \operatorname{Pic}(R)) \stackrel{\varphi_{6}}{\rightarrow} H^{3}(G, \mathcal{U}(R))$.

Proof. When the action $\alpha$ is global we have $R^{\alpha}=R^{G}$ and $D_{g}=R$ for any $g \in G$. The latter implies $H^{i}(G, \alpha, R)=H^{i}(G, \mathcal{U}(R))$, for $1 \leq i \leq 3$. Moreover $\operatorname{PicS}(R)^{\alpha^{*}}=$ $\operatorname{PicS}(R)^{G}=\left\{[E] \in \mathbf{P i c S}(R) \mid E_{g} \cong E\right.$ for any $\left.g \in G\right\}$ and then $\operatorname{PicS}(R)^{\alpha^{*}} \cap \operatorname{Pic}(R)=$ $\operatorname{Pic}(R)^{G}$. Finally,

$$
\begin{aligned}
\operatorname{cls}(f) \in H^{1}\left(G, \alpha^{*}, \mathbf{P i c S}(R)\right) & \Leftrightarrow f \in Z^{1}\left(G, \alpha^{*}, \mathbf{P i c S}(R)\right) \& f(g) \in \mathbf{P i c}\left(D_{g}\right), \forall g \in G . \\
& \Leftrightarrow f \in Z^{1}\left(G, \alpha^{*}, \mathbf{P i c S}(R)\right) \& f(g) \in \mathbf{P i c}(R), \forall g \in G . \\
& \Leftrightarrow f \in Z^{1}(G, \mathbf{P i c}(R)),
\end{aligned}
$$

so that $H^{1}\left(G, \alpha^{*}, \mathbf{P i c S}(R)\right)=H^{1}(G, \mathbf{P i c}(R))$, and we obtain the sequence as given in $[5]$.

Remark 7.11. The fact that $\varphi_{6}(\operatorname{cls}(f))=\operatorname{cls}(\omega) \in H^{3}(G, \alpha, R)$ for every 1-cocycle $f \in Z^{1}\left(G, \alpha^{*}, \operatorname{PicS}(R)\right)$ was proved in $\left[17\right.$, Section 6.1] by showing that $\omega_{\mathfrak{p}}$ is a 3coboundary for any prime $\mathfrak{p} \in \operatorname{Spec}\left(\mathrm{R}^{\alpha}\right)$. Thus it is reasonable to say that a partial cocycle $\omega \in Z^{n}(G, \alpha, R)$ is a local coboundary if for any $\mathfrak{p} \in \operatorname{Spec}\left(\mathrm{R}^{\alpha}\right)$ there exists $\rho \in C^{n-1}\left(G, \alpha_{\mathfrak{p}}, R_{\mathfrak{p}}\right)$ such that $\omega\left(g_{1}, \ldots, g_{n}\right)_{\mathfrak{p}}=\left(\delta^{n-1} \rho\right)\left(g_{1}, \ldots, g_{n}\right)$, where $\alpha_{\mathfrak{p}}$ is the partial action of $G$ on $R_{\mathfrak{p}}$ which corresponds to $\alpha$. Then we may call the cohomology class of $\omega \in Z^{n}(G, \alpha, R)$ locally trivial if $\omega$ is a local coboundary, and we denote by $H_{\mathrm{lt}}^{n}(G, \alpha, R)$ the subgroup of $H^{n}(G, \alpha, R)$ which consists of the locally trivial cohomology classes. With this notation we have that $\operatorname{Im} \varphi_{6} \subseteq H_{\mathrm{lt}}^{3}(G, \alpha, R)$.

\section{REFERENCES}

[1] M. Auslander, O. Goldman, The Brauer group of a commutative ring Trans. Amer. Math. Soc. (1960) 52: 367-409.

[2] G. Azumaya, Completely faithful modules and self injective rings Nagoya Math. (1966) 27: 697-708.

[3] E. Batista, Partial actions: What they are and why we care, Bull. Belg. Math. Soc. - Simon Stevin (2017) 24: (1), 35-71.

[4] E. Batista, A. D. M. Mortari, M. M. Teixeira, Cohomology for partial actions of Hopf algebras, J. Algebra, 528 (2019), 339-380.

[5] S.U. Chase, D.K. Harrison, A. Rosenberg, Galois theory and Galois cohomology of commutative rings, Mem. Amer. Math. Soc. 52 (1965) 15-33. 
[6] S. U. Chase, A. Rosenberg, Amitsur cohomology and the Brauer group, Mem. Am. Math. Soc. 52 (1965) 34-79.

[7] F. DeMeyer, E. Ingraham, Separable algebras over commutative rings, Lect. notes in math., 181, Springer (1971).

[8] M. Dokuchaev, Partial actions: a survey, Contemp. Math., 537 (2011), 173-184.

[9] M. Dokuchaev, Recent developments around partial actions, São Paulo J. Math. Sci. (2019) 13: (1), 195-247.

[10] M. Dokuchaev, R. Exel, Associativity of crossed products by partial actions, enveloping actions and partial representations. Trans. Amer. Math. Soc. (2005) 357: 1931-1952.

[11] M. Dokuchaev, R. Exel, J. J. Simón, Crossed products by twisted partial actions and graded algebras, J. Algebra (2008) 320: 3278-3310.

[12] M. Dokuchaev, R. Exel, P. Piccione, Partial representations and partial group algebras, J. Algebra, 226 (1) (2000), 505-532.

[13] M. Dokuchaev, M. Ferrero, A. Paques, Partial actions and Galois theory, J. Pure Appl. Algebra (2007) 208: 77-87.

[14] M. Dokuchaev, M. Khrypchenko, Partial cohomology of groups, J. Algebra (2015) 427: 142-182.

[15] M. Dokuchaev, M. Khrypchenko, Twisted partial actions and extensions of semilattices of groups by groups, Int. J. Algebra Comput., 27, (7) (2017), 887-933.

[16] M. Dokuchaev, M. Khrypchenko, Partial cohomology of groups and extensions of semilattices of abelian groups, J. Pure Appl. Algebra, 222, (2018), 2897-2930.

[17] M. Dokuchaev, A. Paques, H. Pinedo, Partial Galois cohomology, extensions of the Picard group and related homomorphisms, Quart. J. Math. 70, (2019), (2), 737-766, expanded version in arXiv:1804.03762.

[18] M. Dokuchaev, N. Sambonet, Schur's theory for partial projective representations, Israel J. Math. (to appear), arXiv:1711.06739 (2017).

[19] R. Exel, Twisted partial actions: a classification of regular $C^{*}$-algebraic bundles, Proc. London Math. Soc., 74 (1997), (3), 417-443.

[20] R. Exel, Partial Dynamical Systems, Fell Bundles and Applications, Mathematical Surveys and Monographs, vol. 224, American Mathematical Society, 2017.

[21] M. Ferrero, Partial actions of groups on algebras, a survey, São Paulo J. Math. Sci. 3 (2009), (1), 95-107.

[22] T. Ford, Separable Algebras, Am. Math. Soc. (2017) 187.

[23] T. Kanzaki, On generalized crossed product and Brauer group, Osaka J. Math (1968) 5: 175-188.

[24] M. Kennedy, C. Schafhauser, Noncommutative boundaries and the ideal structure of reduced crossed products, Duke Math. J. (to appear), arXiv:1710.02200 (2017).

[25] T. Y. Lam, Lectures on Modules and Rings, $2^{\text {nd }}$ Edition Springer (1998).

[26] T. Y. Lam, A first Course in Noncommutative Rings, $2^{\text {nd }}$ Edition Springer (1990).

[27] A. Paques, A. Sant'Ana, When is a crossed product by a twisted partial action Azumaya?, Comm. Algebra (2010) 38: 1093-1103.

[28] H. Pinedo, Partial projective representations and the partial Schur multiplier: a survey, Boletín de Matemáticas, 22 (2015), (2), 167-175. 
Instituto de Matemática e Estatística, Universidade de São Paulo, Rua do Matão, 1010, 05508-090 SÃo Paulo, SP, Brasil.

E-mail address: dokucha@ime.usp.br

Instituto de Matemática Universidade Federal do Rio Grande do Sul, Avenida Bento Gonçalves 9500, 91509-900 Porto Alegre, RS, Brasil.

E-mail address: paques@mat.ufrgs.br

Escuela de Matemáticas, Universidad Industrial de Santander, Cra 27 calle 9, Edificio Camilo Torres, Bucaramanga, Colombia.

E-mail address: hpinedot@uis.edu.co

Unidade Acadêmica de Matemática, Universidade Federal de Campina Grande, Avenida Aprígio Veloso, 882, 58429-900, Campina Grande, PB, Brasil.

E-mail address: itailma@mat.ufcg.edu.br 